ver. 29 Nov. 2004

\title{
DUST IN THE PHOTOSPHERIC ENVIRONMENT III. A FUNDAMENTAL ELEMENT IN THE CHARACTERIZATION OF ULTRACOOL DWARFS
}

\author{
TAKASHI TSUJI \\ Institute of Astronomy, School of Science, The University of Tokyo \\ 2-21-1, Osawa, Mitaka, Tokyo, 181-0015, Japan \\ ttsuji@ioa.s.u-tokyo.ac.jp
}

\begin{abstract}
Recent photometry of $\mathrm{L}$ and $\mathrm{T}$ dwarfs revealed that the infrared colors show a large variation at a given effective temperature and, within the framework of our Unified Cloudy Model (UCM), this result can be interpreted as due to a variation of the critical temperature $\left(T_{\mathrm{cr}}\right)$ which is essentially a measure of the thickness of the dust cloud. In our previous applications of the UCMs, we assumed that $T_{\text {cr }}$ is constant at about $1800 \mathrm{~K}$ in all the $\mathrm{L}$ and $\mathrm{T}$ dwarfs but, in view of the new observing result, we now allow $T_{\mathrm{cr}}$ to vary between the surface temperature $\left(T_{0}\right)$ and the condensation temperature $\left(T_{\text {cond }}\right)$ at given $T_{\text {eff }}$ and $\log g$. Then, the two-color diagram and color-magnitude diagram can be well explained as the effect of $T_{\text {eff }}, \log g$, and $T_{\text {cr }}$, but not by the effect of $T_{\text {eff }}$ and $\log g$ alone. This result implies that $T_{\text {cr }}$ will be one of the important parameters needed for characterization of dusty dwarfs. The effects of $T_{\text {eff }}$ and $T_{\text {cr }}$ on individual spectra, however, are difficult to discriminate, since changing of $T_{\text {eff }}$ at a fixed $T_{\text {cr }}$ on one hand and changing of $T_{\text {cr }}$ at a fixed $T_{\text {eff }}$ on the other essentially have the same effect on the spectra. We show that the degeneracy of $T_{\text {eff }}$ and $T_{\text {cr }}$ can be removed to some extent by the analysis of the spectral energy distribution (SED) on an absolute scale. The reanalysis of a selected sample of spectra revealed that the L-T spectral sequence may not necessarily be a sequence of $T_{\text {eff }}$, but may reflect a change of the thickness of the dust cloud, represented by $T_{\text {cr }}$ in our UCM. Although this unexpected result is based on a limited sample, an odd "brightening" of the absolute $J$ magnitudes plotted against the L-T spectral types may also be a manifestation that the L-T spectral sequence is not necessarily a temperature sequence. This is because $M_{\text {bol }}$ based
\end{abstract}


on the same photometry data also shows a similar "brightening". Then, the " $J$ brightening" may not be due to any atmospheric effect and hence should not be a problem to be solved by model atmospheres including the UCMs. Thus, almost all the available observed data are reasonably well interpreted with the UCMs, whose full capability emerges once $T_{\text {cr }}$ is introduced as the fifth parameter in addition to the usual four parameters (i.e., chemical composition, $T_{\text {eff }}, \log g$, and micro-turbulent velocity) needed to characterize stellar spectra in general.

Subject headings: molecular processes — stars: atmospheres — stars: fundamental parameters — stars: late-type — stars: low-mass, brown dwarfs -

\section{INTRODUCTION}

In any branch of natural science, classification of objects to study is a fundamental step, from which next developments will be conceived and undertaken. Thus, it is quite natural that the spectral classification of ultracool dwarfs including brown dwarfs has been initiated as soon as some dozens of dwarfs cooler than type M have been discovered in the late 1990's. A new spectral class L was assigned to these objects, whose prototype is GD165b (Becklin \& Zuckermann 1988), and spectral subclass was defined by considering various spectral features in the optical spectra (Kirkpatrick et al. 1999; Martín et al. 1999). Soon the near infrared spectra are used for the spectral classification (Reid et al. 2001; Testi et al. 2001). It did not take long before a considerable number of cool brown dwarfs, whose prototype is Gl229b (Nakajima et al. 1995), have been discovered, and an attempt to classify them under a new spectral class T was undertaken (Burgasser et al. 2002a). Finally, a unified classification scheme of $\mathrm{L}$ and $\mathrm{T}$ types was proposed with the use of the near infrared spectra (Geballe et al. 2002). Now, a question is what the $\mathrm{L}$ and T types mean.

The stellar spectral classification, which has the present form at the beginning of the 20th century, was done on purely empirical basis at first. It was later interpreted as a temperature sequence and further developed to the two dimensional classification including the luminosity effect. The physical basis of the present two dimensional stellar spectral classification such as MK system is quite clear: The basic stellar properties represented by luminosity $L$ and radius $R$, from which $T_{\text {eff }}$ and $\log g$ are defined, can be determined uniquely as a function of time once the initial mass and chemical composition are given (Vogt-Russell theorem; e.g. Chandrasekhar 1939). Thus any object on the HR diagram has its corresponding spectral type. Later it was recognized that the characterization of stellar spectra requires an additional parameter referred to as the micro-turbulent velocity (Struve \& Elvey 1934) which, however, is more difficult to predict for individual objects. 
A problem is if the case of $\mathrm{L}$ and $\mathrm{T}$ types can be a simple extension of this paradigm. A new feature of $\mathrm{L}$ and $\mathrm{T}$ dwarfs is that dust forms in their photosphere, and we proposed that the L-T spectral sequence can be interpreted with a model which we referred to as the Unified Cloudy Model (Tsuji 2001): In this UCM, dust forms at the condensation temperature $T_{\text {cond }}$, but soon grows to be too large at a slightly lower temperature which was referred to as the critical temperature $T_{\text {cr }}$, and segregates from the gaseous mixtures. Thus a dust cloud forms in the layer where $T_{\text {cr }} \lesssim T \lesssim T_{\text {cond }}$. In this model, $T_{\text {cond }}$ is essentially determined by the thermodynamical data and thus well predictable for the basic parameters such as $T_{\text {eff }}, \log g$, and metallicity. At first, we also thought that $T_{\text {cr }}$ may in principle have a causal relation to the basic parameters even if we do not yet know the exact relation. Then, we assumed a constant value for $T_{\text {cr }}$ for simplicity, and estimated it empirically. Since $T_{\text {cr }}$ is a measure of the thickness of the dust cloud, which has a direct effect on the infrared colors, we estimated $T_{\text {cr }}$ to be about $1800 \mathrm{~K}$ from the red limit of $J-K$ (Tsuji 2002; hereafter be referred to as Paper I). Then it was possible to interpret the spectral sequence from $\mathrm{L}$ to $\mathrm{T}$ as a temperature sequence (Tsuji, Nakajima, \& Yanagisawa 2004; hereafter be referred to as Paper II).

Recent progress in observations, however, casts some doubts as to whether if the L-T spectral types are simple extension of the stellar spectral types to the cooler temperatures. Especially, the effective temperatures based on the bolometric luminosities determined from the recent astrometry (Vrba et al. 2004) and photometry extended to $L^{\prime}$ and $M^{\prime}$ bands (Golimowski et al. 2004) appeared to be nearly constant between middle L and early $\mathrm{T}$ types (Vrba et al. 2004; Golimowski et al. 2004). This fact suggests that the L-T spectral sequence may not necessarily be a temperature sequence and that the spectral sequence is controlled by parameter(s) other than $T_{\text {eff. }}$ Also, it appears that the infrared colors extended to a large sample of $\mathrm{L}$ and $\mathrm{T}$ dwarfs (Knapp et al. 2004) show a large variation especially if they are plotted against $T_{\text {eff }}$. This fact implies that $T_{\text {cr }}$, which is directly related to the infrared colors as noted above, cannot be a constant throughout $\mathrm{L}$ and $\mathrm{T}$ dwarfs as assumed in our Papers I and II, but should show large changes independently of the basic stellar properties such as $T_{\text {eff }}$ and $\log g$ (see Sect.2.1 as for details). Hence any conclusion based on this assumption must be reconsidered.

In our UCMs, the property of the dust cloud is represented by a single parameter, $T_{\mathrm{cr}}$, which is a measure of the thickness of the dust cloud. This is essentially equivalent to have specified the dust column density in the cloud for the distribution of dust grains given by the static model photosphere. The cloud consists of grains smaller than the critical radius (Paper I) and the grain size is assumed to be small enough to be in the Rayleigh regime (i.e. $r_{\mathrm{gr}}<<\lambda$ ). No other details of the cloud is specified in the present UCMs. If we are to specify some details of the clouds, we must make some, possibly ad hoc, assumptions, since we do not know the details how clouds are formed in the photospheres of cool dwarfs at 
present. It is to be remembered that $T_{\mathrm{cr}}$ is an empirical parameter and it is not derived from any particular model of cloud formation. The new feature that the thickness of the dust cloud changes somewhat accidentally implies that the cloud formation may take place under chaotic condition and/or may already be a meteorological phenomenon. Since theoretical prediction of an accidental event is more difficult, it may be useful to have emphasized an empirical approach before more serious effort of detailed modeling of the cloud formation will be done. It is to be remembered that a purpose of modeling is to describe complicated astronomical phenomenon by a simple picture as far as possible at first, and our UCM is presently at this stage.

In this paper, we hope to examine to what extent our simple model could explain the available observed data on $\mathrm{L}$ and $\mathrm{T}$ dwarfs. First, we will show that most observations such as colors and magnitudes (Sect.2) as well as spectra (Sect.3) can be accounted for reasonably well by our UCMs if we assume that the critical temperature $T_{\text {cr }}$ varies between the surface temperature $\left(T_{0}\right)$ and the condensation temperature $\left(T_{\text {cond }}\right)$ at fixed $T_{\text {eff }}$ and $\log g$. This fact confirms that the thickness of the dust cloud, or $T_{\text {cr }}$ in our UCM, is an important parameter in interpreting the observed data of dusty dwarfs. Next, we discuss how the basic properties including effective temperature, luminosity, and spectral classification can be interpreted by the applications of the UCMs with the variable $T_{\text {cr }}$ (Sect.4). However, some conclusions

appear to be quite unexpected: For example, it is shown that the L-T spectral sequence is not necessarily a sequence of $T_{\text {eff }}$ but $T_{\text {cr }}$ plays dominant role at least between middle $\mathrm{L}$ and early $\mathrm{T}$ types. This is because both of $T_{\text {eff }}$ and $T_{\text {cr }}$ have significant effect on the dust column density in the observable photosphere, and $T_{\text {cr }}$ is sometimes more important than $T_{\text {eff }}$ in characterization of the photosphere of dusty dwarfs. In our formulation of the UCMs, this new parameter $T_{\text {cr }}$ distinguishes dusty dwarfs from other stars, which are characterized by four parameters, namely, chemical composition, $T_{\text {eff }}, \log g$, and micro-turbulent velocity.

\section{Colors and Magnitudes}

Recent infrared photometry revealed that infrared colors plotted against $T_{\text {eff }}$ show a large variation, and this fact implies that the thickness of the dust cloud should be changing independently of $T_{\text {eff }}$. In our UCMs, the critical temperature $T_{\text {cr }}$ is introduced as a measure of the thickness of the dust cloud, but it was assumed that $T_{\text {cr }}$ is constant throughout $\mathrm{L}$ and T dwarfs for simplicity in Papers I and II. This is partly because the scatters of the infrared colors plotted against the spectral type appeared to be not so large as those plotted against $T_{\text {eff }}$. In view of the new feature, however, we think it an over-simplification to have assumed a unique value for $T_{\mathrm{cr}}$. Furthermore, this assumption might have suppressed the potentiality 
that the UCMs could be able to have if they are applied more properly. We now regard $T_{\text {cr }}$ as a free parameter to be variable between the condensation and surface temperatures. Then, we demonstrate that such purely empirical data as the two-colors diagram and colormagnitude $(\mathrm{CM})$ diagram of $\mathrm{L}$ and $\mathrm{T}$ dwarfs can be explained with the three parameters, $T_{\text {eff }}, \log g$, and $T_{\text {cr }}$ while not at all by the two parameters, $T_{\text {eff }}$ and $\log g$.

\subsection{Infrared Colors and the Critical Temperature}

Infrared colors plotted against L - T spectral types first show reddening from early to late $\mathrm{L}$ types and, after a reversal at late $\mathrm{L}$, show bluing from early to late $\mathrm{T}$ types (e.g. Leggett et al. 2002). Recent progress in observations revealed some new features: First, thanks to the extended parallax data (Vrba et al. 2004) and bolometric corrections based on the $L^{\prime}$ and $M^{\prime}$ photometry (Golimowski et al. 2004), absolute bolometric luminosities are obtained for a large sample of $\mathrm{L}$ and $\mathrm{T}$ dwarfs. Then empirical effective temperatures can be derived if the radii of $\mathrm{L}$ and $\mathrm{T}$ dwarfs can be known. For this purpose, Vrba et al. (2004) assumed a constant radius of $0.9 R_{\text {Jupiter }}$ while Golimowski et al. (2004) applied the radii based on evolutionary models. The resulting values of $T_{\text {eff }}$ at 3 Gyrs by Golimowski et al. (2004) mostly agree with those by Vrba et al. (2004) (see Sect.4.2), and this is what can be expected since the parallax data and bolometric corrections are mostly the same in the two works. We plotted the observed colors on the MKO system (Knapp et al. 2004) against the empirical values of $T_{\text {eff }}$ (Vrba et al. 2004) rather than against the $\mathrm{L}$ - T spectral types in

Fig. 1, where $T_{\text {eff }}$ values based on the bolometric luminosities are shown by the large circles and those based on the estimations with the $T_{\text {eff }}-\mathrm{Sp}$. Type relation by the small circles. In Fig. 1, L and $\mathrm{T}$ dwarfs are shown by the filled and open circles, respectively, and it appears that the transition from $\mathrm{L}$ to $\mathrm{T}$ types takes place at $T_{\mathrm{eff}} \approx 1400 \pm 100 \mathrm{~K}$. It is remarkable in Fig. 1 that the scattering is very larger especially at around $T_{\text {eff }} \approx 1400 \mathrm{~K}$. A preliminary result that the infrared colors such as $J-K$ plotted against $T_{\text {eff }}$ (and not against the $\mathrm{L}-\mathrm{T}$ types) show a drastic change at the $\mathrm{L}$ - T transition was already noted by us (Tsuji 2005), independently by Marley, Cushing, \& Saumon (2005), and also by Leggett et al. (2005).

Although it was known that the scatters of the infrared colors plots against the spectral type are rather large in L dwarfs (e.g. Fig. 3 of Knapp et al. 2004), the plots of the same colors against effective temperature revealed that the scatters are much larger especially at around the $\mathrm{L}-\mathrm{T}$ transition. It is remarkable that the plots of the same infrared colors against the spectral type and those against the effective temperature are so different. This means that an implicit assumption that the $\mathrm{L}$ - T spectral sequence is a temperature sequence may not necessarily be correct, even though this might be a rather natural assumption in view 
of the smooth change of the infrared colors plotted against the spectral types. In fact, this conventional assumption now appears to be not consistent with the recent empirical effective temperature determinations (e.g. Golimowski et al. 2004; Vrba et al. 2004).

The modest scatters of the infrared colors plotted against the L-T spectral were already interpreted as due to variations in the altitudes, spatial distributions, and thicknesses of the clouds (Knapp et al. 2004). The scatter of the near infrared colors at the same L spectral types was previously noted on the 2MASS data by Stephens (2003) and interpreted as due to the change of the cloud opacity which depends on the sedimentation efficiency $f_{\text {sed }}$ of the cloud model of Ackerman \& Marley (2001). In our UCM, the thickness of the dust cloud is represented by a parameter referred to as the critical temperature $T_{\text {cr }}$ (Tsuji 2002), but we assumed it to be a constant for simplicity in our previous applications of UCMs (e.g. Tsuji \& Nakajima 2003; Tsuji et al. 2004). This assumption, however, should be reconsidered in view of the much larger scatter of the infrared colors as revealed in Fig. 1.

For this purpose, we first examine the effect of gravity and plot the predicted infrared colors based on the UCMs with $\log g=4.5,5.0$ and 5.5 in Fig. $1 \mathrm{a}$ ( $T_{\text {cr }}=1800 \mathrm{~K}$ throughout). We applied the filter response functions (Tokunaga, Simon, \& Vaccatok 2002) to the predicted fluxes based on the case I (band model) opacities for methane (see Paper II as for detail). Inspection of Fig. 1a reveals that some scatters of the observed infrared colors can be explained as the gravity effect (e.g. $J-K$ at $T_{\text {eff }} \gtrsim 1600 \mathrm{~K}$ and $H-K$ at $T_{\text {eff }} \lesssim 1300 \mathrm{~K}$ ) and, for this reason, we previously thought that the scatter of $J-K$ can be explained by the gravity effect (Paper II). Such a gravity effect on the infrared colors was also discussed by Knapp et al. (2004) on the basis of the cloudy models by Marley et al. (2002). However, it is clear in Fig. 1b that the large variations of the infrared colors near the $\mathrm{L}-\mathrm{T}$ transition cannot be explained by the gravity effect. It is to be noted that the large variations of the infrared colors at $T_{\text {eff }} \approx 1400 \mathrm{~K}$ has not been recognized before, and it is the smaller variations of the infrared colors plotted against the $\mathrm{L}$ - T spectral types that were thought to be interpreted as the gravity effect in our Paper II. Now the situation is completely different because of the drastic changes of the infrared colors at $T_{\text {eff }} \approx 1400 \mathrm{~K}$, and we must now recognize that the large scatter of the infrared colors near the $\mathrm{L}-\mathrm{T}$ transition cannot be explained by the gravity effect.

Next, we overlaid the predicted colors based on the UCMs with $T_{\mathrm{cr}}=1700,1800,1900 \mathrm{~K}$, and $T_{\text {cond }}$ in Fig. $1 \mathrm{~b}(\log g=5.0$ throughout). Previously, we interpreted that the scatters of the colors at their red limit in late $\mathrm{L}$ dwarfs can roughly be explained by $T_{\mathrm{cr}}=1800 \pm 100 \mathrm{~K}$. For this reason, we assumed that $T_{\text {cr }} \approx 1800 \mathrm{~K}$ may apply to all the cool dwarfs from $\mathrm{L}$ to $\mathrm{T}$ throughout in our previous applications of the UCMs (Papers I and II). However, this is clearly an over-simplification in view of the larger variation around the $\mathrm{L}-\mathrm{T}$ transition at 
$T_{\text {eff }} \approx 1400, \mathrm{~K}$ (Fig. 1 ), which has not been recognized before when the infrared colors were plotted against the L - T spectral type. Now, to explain the large variations of the infrared colors near the $\mathrm{L}$ - $\mathrm{T}$ transition, we must assume from Fig. $1 \mathrm{~b}$ that $T_{\text {cr }}$ in our UCM, or the thickness of the dust cloud, should be different at a given $T_{\text {eff }}{ }^{1}$. Moreover, the variation of the thickness of the dust cloud should be quite drastic such that $T_{\text {cr }}$ changes from $T_{\text {cond }}$ to below $1700 \mathrm{~K}$ in our UCM. Note that the case of $T_{\text {cr }} \approx T_{\text {cond }}$ implies that the dust cloud effectively disappears.

In the UCMs, $T_{\text {cond }}$ is predictable based on the thermochemical data and hence it is well defined for given $T_{\text {eff }}$ and $\log g$. In general, $T_{\text {cond }}$ is higher at at higher gravities and/or at lower effective temperatures, because of the higher gas pressures in these cases. For example, $T_{\text {cond }}$ values of iron are 1900 and $2200 \mathrm{~K}$ for models of $T_{\text {eff }}=1800$ and $1000 \mathrm{~K}$, respectively $(\log g=5.0$ throughout; see Fig. 3 in Paper I). We have already considered a possibility of variable $T_{\text {cr }}$ but in such a way that $T_{\text {cr }}$ depends on $T_{\text {eff }}$ and $\log g$. In fact, the thickness of the cloud will be very thin in early $\mathrm{L}$ dwarfs for $T_{\text {cr }} \approx 1800 \mathrm{~K}$, since $T_{\text {cond }}$ will already be close to $1800 \mathrm{~K}$ for the models of relatively high $T_{\text {eff }}$, and we considered a possibility that $T_{\text {cr }}$ may be lower for the models of high $T_{\text {eff }}$ (Paper I $)^{2}$. With such a possibility in mind, we have prepared the UCMs with several values of $T_{\mathrm{cr}}$. However, the fact realized in nature turns out to be beyond what we have imagined: The variation of $T_{\text {cr }}$ appears to be independent of $T_{\text {eff }}$ and $\log g$ (Figs. 1a \& b), and to be quite large, but the origin of the variation cannot be understood at all.

The fact that the transition from $\mathrm{L}$ to $\mathrm{T}$ takes place at $T_{\text {eff }} \approx 1400 \mathrm{~K}$ implies that $T_{\text {eff }}$ is an important factor in the $\mathrm{L}-\mathrm{T}$ transition, but it is also clear that another effect plays decisive role. Within the framework of our UCMs, this effect is identified with $T_{\text {cr }}$ which is a measure of the thickness of the dust cloud. It is as if something might have happened at $T_{\text {eff }} \approx 1400 \mathrm{~K}$ to induce the change of the thickness of the cloud in such a way that $\mathrm{L}$ dwarfs with the lower values of $T_{\text {cr }}$ evolve to $\mathrm{T}$ dwarfs with the higher values of $T_{\text {cr }}$. The rapid and almost discontinuous change of the infrared colors at the $\mathrm{L}$ - $\mathrm{T}$ transition in a very small $T_{\text {eff }}$ range is also clearly illustrated in Fig. 5 of Marley et al. (2005). Since the L - T transition

\footnotetext{
${ }^{1}$ It is to be noted that the thickness of the cloud, or more properly, the dust column density in the observable photosphere, is not necessarily the same even if $T_{\text {cr }}$ is fixed, since $T_{\text {cond }}$ shows continuous changes through L to T dwarfs (see Fig. 3 in Paper I). In fact, this may be the reason why infrared colors, largely controlled by the dust extinction, change with $T_{\text {eff }}$. However, the change of the dust column density due to $T_{\text {cr }}$ itself introduced here is independent of $T_{\text {eff }}$ and of different nature.

${ }^{2}$ In the early $\mathrm{L}$ dwarfs, observed colors appear to be redder than the predicted ones for any value of $T_{\text {cr }}$ in Fig. 1. This result may not necessarily be related to dust, but may be due to some other problems such as of the gaseous opacities.
} 
is usually discussed in connection with the CM diagrams, we will return to this subject in Sect. 2.3.

A further complication is that $T_{\mathrm{cr}}$ and $T_{\text {eff }}$ have nearly the same effect on the infrared colors. For example, we interpreted the reddening of the infrared colors in L dwarfs as due to the increase of the dust column density in the observable photosphere with the decreasing $T_{\text {eff }}$. But the dust column density will also increase with decreasing $T_{\text {cr }}$ even at the fixed $T_{\text {eff }}$. Thus, $T_{\text {eff }}$ and $T_{\text {cr }}$ have the same effect on the infrared colors. In Fig. 1b, it was possible to separate the effect of $T_{\text {eff }}$ and $T_{\text {cr }}$, and this is because $T_{\text {eff }}$ was known by other method in this case. Generally, it is difficult to remove the degeneracy of $T_{\text {eff }}$ and $T_{\text {cr }}$ unless an independent information on one of the two can be available. This problem will further be discussed in Sect.3.

\subsection{Two-Colors Diagram}

We plotted the observed $J-H$ against $H-K$ on the MKO system (Knapp et al. 2004) in Fig. 2a, where $\mathrm{L}$ and $\mathrm{T}$ dwarfs are shown by filled and open circles, respectively. We overlaid the predicted colors based on the UCMs with the three values of $\log g=4.5,5.0$ and 5.5, but with the constant value of $T_{\mathrm{cr}}=1800 \mathrm{~K}$ throughout. The effect of gravity is minor in L and early $\mathrm{T}$ dwarfs, and the observed scatters of the colors cannot be explained as the gravity effect for these dwarfs. On the other hand, the scatters of the observed colors in middle and late $\mathrm{T}$ dwarfs are well explained as the gravity effect. Thus we agree as to the gravity effect in T dwarfs with Knapp et al. (2004) who applied the models of Marley et al. (2002). Note that our UCMs cover the range of $T_{\text {eff }}$ between 700 and $2600 \mathrm{~K}$. Thus an object at the lower left corner 2MASS J0937+2931 (T6p) may have $\log g$ somewhat lower than 5.0 and $T_{\text {eff }}$ somewhat lower than $700 \mathrm{~K}$ from the colors alone if metallicity is normal.

In Fig. 2b, we overlaid the predicted colors based on the UCMs with $T_{\text {cr }}=T_{0}$ (case B), $1700,1800,1900 \mathrm{~K}$ and $T_{\text {cond }}$ (case $\left.\mathrm{C}\right)$ on the same observed data as in Fig. $2 \mathrm{a}(\log g=5.0$ throughout). It is confirmed that most of the colors of $\mathrm{L}$ and early $\mathrm{T}$ dwarfs can be explained by changing the $T_{\text {cr }}$ values from $T_{\text {cond }}$ (case C) to $T_{0}$ (case B). Several early $\mathrm{T}$ dwarfs are found in the region of $1900 \mathrm{~K}<T_{\mathrm{cr}}<T_{\text {cond }}$ and it is interesting if this fact implies that the dust cloud is really very thin in the early $\mathrm{T}$ dwarfs. Few objects are found on the right side

of the extreme case of fully dusty models (case B). The effect of $T_{\text {cr }}$ is very small in middle and late $\mathrm{T}$ dwarfs and the scatters of the observed colors are mostly explained as the gravity effect as noted already.

To summarize, almost all the data points in the observed $(J-H, H-K)$ diagram could 
be explained by changing the three parameters $T_{\mathrm{eff}}, \log g$, and $T_{\mathrm{cr}}$ in appropriate ranges in our UCMs. It should be noted that the role of $T_{\text {cr }}$ is essential (Fig. $2 \mathrm{~b}$ ) while $T_{\text {eff }}$ and $\log g$ alone could not explain the two-colors diagram (Fig. 2a).

\subsection{Color-Magnitude Diagram}

Recent progress of astrometry and photometry of ultracool stars finally made it possible to compare CM diagrams with the theoretical evolutionary tracks of substellar objects. Our previous attempt on the $\left(M_{J}, J-K\right)$ diagram (Tsuji \& Nakajima 2003), however, was not fully successful, but received some criticisms. First, some early $\mathrm{T}$ dwarfs that show the so-called "J-brightening" was interpreted as the very young low-mass objects, but such a hypothesis does not necessarily find observational support. The so-called "J-brightening" seen on the plot of $M_{J}$ vs. Sp. Types may be an artifact of the L-T spectral sequence not representing a temperature sequence as will be shown in Sect.4.1, but the "J-brightening" on the CM diagram still remains to be explained. Second, some late L dwarfs are too faint to be explained by our previous analysis.

We must point out that our previous attempt was based on an assumption of a uniform value of $T_{\text {cr }} \approx 1800 \mathrm{~K}$ throughout the $\mathrm{L}-\mathrm{T}$ dwarfs, but this assumption can no longer be supported as noted in Sects.2.1 and 2.2. We then examine the effect of changing $T_{\text {cr }}$ on the CM diagram for the three evolutionary tracks of $M=10,42$, and $70 M_{\text {Jupiter }}$ (Burrows et al. 1997) separately, and compared the results with the observed data on the MKO system (Knapp et al. 2004). We evaluate $J-K$ and $B C_{\mathrm{J}}$ based on the UCMs with different values of $T_{\text {cr }}{ }^{3}$, using the filter response functions of the MKO system (Tokunaga et al. 2002), and converted $\left(T_{\text {eff }}, M_{\text {bol }}\right)$ to $\left(J-K, M_{J}\right)$ with the use of these results. First, the case of $M=10 M_{\text {Jupiter }}$ are shown in Fig. $3 \mathrm{a}$ for $T_{\text {cr }}=1700,1800,1900 \mathrm{~K}$ and $T_{\text {cond }}$ (case $\mathrm{C}$ ) . The agreement between observed and predicted loci is generally poor except for early and middle $\mathrm{L}$ dwarfs. This fact can be interpreted that the low-mass brown dwarfs are more difficult to observe because of their short lifetimes and thus they may be under-sampled in the presently observed sample of brown dwarfs.

The same analysis is done for the case of $M=42 M_{\text {Jupiter }}$, and the results are shown in Fig. 3b. In this case, most of the observed data points, except for late $\mathrm{L}$ dwarfs, are covered by the predicted tracks with the values of $T_{\text {cr }}$ between $1700 \mathrm{~K}$ and $T_{\text {cond }}$. The so-called " $J$ brightening" of some early $\mathrm{T}$ dwarfs are now explained by the models with very thin clouds

\footnotetext{
${ }^{3}$ Some examples of the values of $B C_{\mathrm{J}}$ (CIT system) for several values of $T_{\mathrm{cr}}$ are given in Fig. 2 of Tsuji \& Nakajima (2003).
} 
or with no cloud as the limiting case of reduced cloud thickness. This result is consistent with Fig. 1b suggesting that $T_{\text {cr }}$ should be quite high in some early $\mathrm{T}$ dwarfs, and with the suggestion from the two-colors diagram discussed in Sect.2.2 that the thickness of the cloud may be quite thin (i.e., $T_{\text {cr }}>1900 \mathrm{~K}$ ) in early T dwarfs. Finally, the case of $M=70 M_{\text {Jupiter }}$ is shown in Fig. 3c, and now the very low luminosities of some late L dwarfs are explained by the models with the thick cloud (i.e. $T_{\text {cr }} \approx 1700 \mathrm{~K}$ ). As to the very faint $\mathrm{L}$ dwarfs slightly below the predicted track for $T_{\text {cr }}=1700 \mathrm{~K}$, possibilities of the lower $T_{\text {cr }}$ and/or higher masses may be considered.

Summarizing, the observed $\left(M_{J}, J-K\right)$ diagram can reasonable be well reproduced with the evolutionary tracks by Burrows et al. (1997) ${ }^{4}$ and the UCMs in which the parameter $T_{\text {cr }}$ is allowed to change by several hundreds Kelvin. The rapid bluing and brightening at the transition from $\mathrm{L}$ to $\mathrm{T}$ can be explained primarily by the immersion of the dust cloud from the optically thin to thick region at about $T_{\text {eff }} \approx 1400 \pm 100 \mathrm{~K}$ but, at the same time, the thickness of the dust cloud should decrease as suggested by the increase of $T_{\text {cr }}$ (Fig. 1b). Such a change of the thickness of the dust cloud cannot be explained by the known theories of the structure and evolution of brown dwarfs, and probably not yet identified dynamical process must be called for.

In a recent paper, Knapp et al. (2004) pointed out several difficulties in our previous model of the L - T transition (Tsuji \& Nakajima 2003), but we hope that these issues are mostly resolved by our present version of UCMs with the variable cloud thickness. It is to be noted, however, that our UCMs assume a homogeneous cloud throughout. A possibility of clouds with holes has been proposed to explain the rapid transition from $\mathrm{L}$ to $\mathrm{T}$ as well as the "J-brightening" by Burgasser et al. (2002b), and further discussed by Knapp et al. (2004) who referred to it as the "patchy clouds" model. We think that such an inhomogeneity does not necessarily be required at present, even though such a possibility may not be excluded a priori. Also, Knapp et al. (2004) suggested another possibility referred to as the "sudden downpour" model based on the cloudy models of Marley et al. (2002): In this model, L dwarfs first cools at essentially constant sedimentation efficiency $f_{\text {sed }}$ and then $f_{\text {sed }}$ begins to increase from $\approx 3$ to infinity at around $T_{\text {eff }} \approx 1300 \mathrm{~K}$. This rapid increase in $f_{\text {sed }}$ will bring rapid cloud thinning and hence rapid bluing infrared colors. We think that this model is more acceptable than the "patchy clouds" model. In fact, the "sudden downpour" model and our UCM with variable cloud thickness are essentially based on the same concept in that both treatments are to specify the cloud properties with simple model parameters, the sedimentation efficiency $f_{\text {sed }}$ and the critical temperature $T_{\text {cr }}$, respectively. Anyhow, all

\footnotetext{
${ }^{4}$ Also, the use of the isochrones by Chabrier et al. (2000) will result in the similar conclusion as can be inferred from our previous result (Tsuji \& Nakajima 2003).
} 
these models including "sudden downpour" model, "patchy clouds" model, and our UCM with variable cloud thickness agree that the nature of the clouds shows drastic changes during the $\mathrm{L}-\mathrm{T}$ transition at $T_{\text {eff }} \approx 1400 \pm 100 \mathrm{~K}$, and to identify the mechanism of driving such changes should be a key to realize the transition from $\mathrm{L}$ to $\mathrm{T}$ types.

\section{Infrared Spectra}

In general, stellar spectra can be characterized by the four parameters, namely, chemical composition, $T_{\text {eff }}, \log g$, and micro-turbulent velocity $v_{\text {micro }}$. In the case of dusty dwarfs, however, an additional parameter that specify the nature of the dust cloud had to be specified for properly interpreting the infrared colors and magnitudes (Sect.2) and we introduced $T_{\text {cr }}$ for this purpose. In this paper, we assume the standard composition throughout and metallicity effect will not be considered for simplicity. Also, we assume $v_{\text {micro }}=1 \mathrm{~km} \mathrm{~s}^{-1}$ throughout. Then, the effectively important parameters are $T_{\text {eff }}, \log g$, and $T_{\mathrm{cr}}$, and a problem is how to interpret the spectra of individual objects characterized by the three parameters. Our UCMs are already prepared for such applications in that they are formulated with the critical temperature $T_{\text {cr }}$ as an important ingredient. The introduction of the new free parameter opens a possibility of more flexible analyses of the observed spectra. Our reanalysis reveals that the previous interpretation of the spectral sequence of ultracool dwarfs such as given in Paper II was not correct, and we believe that we arrive at a more correct interpretation in this paper.

\subsection{Degeneracy of the Effective and Critical Temperatures}

With a proper calibration, spectrum observed with linear detectors can be regarded as a spectral energy distribution (SED) apart from an absolute scale. Such a relative SED is usually analyzed by means of the predicted SEDs based on models, but there is a problem as follows in the case of dusty dwarfs: The dust column density in the observable photosphere depend on $T_{\text {eff }}$ through its effect on $T_{\text {cond }}$ on one hand and on $T_{\text {cr }}$ through its direct effect on the thickness of the cloud on the other. Thus the spectra, which depend on the dust column density in the observable photosphere, depend on $T_{\text {eff }}$ as well as on $T_{\text {cr }}$. Then, the effects of $T_{\text {eff }}$ and of $T_{\text {cr }}$ cannot easily be separated on the observed spectra as well as on the infrared colors (Sect.2.1). Some examples of such a degeneracy between $T_{\text {eff }}$ and $T_{\text {cr }}$ are given below.

First, the observed spectrum of 2MASS 1711 (L6.5) could be fitted with the predicted one of $T_{\text {eff }}=1800 \mathrm{~K}$ on the assumption of $T_{\text {cr }}=1800 \mathrm{~K}$ (Fig. 4a) as in Paper II. However, it 
is more likely that $T_{\text {cr }}<1700 \mathrm{~K}$ from the $J-K$ for this object (Table 1 and Fig. $1 \mathrm{~b}$ ). Then, the same observed spectrum can be fitted with the predicted one of $T_{\text {eff }}=1300 \mathrm{~K}$ on the assumption of $T_{\mathrm{cr}}=1700 \mathrm{~K}$ (Fig. $4 \mathrm{~b}$ ). It is a bit surprising that a difference of only $100 \mathrm{~K}$ in $T_{\text {cr }}$ results in a difference of $500 \mathrm{~K}$ in $T_{\text {eff }}$. But another complication due to dust is coupled here: As can be known by the fact that the same infrared color corresponds to two different values of $T_{\text {eff }}$ (Fig. 1), a very similar SED may result from objects of different $T_{\text {eff }}$ values, one relatively high and the other relatively low, as discussed in Paper II for the case of the L5 dwarf 2MASS 1507 (see its Fig. 13). For this reason, not only $T_{\text {eff }}=1800 \mathrm{~K}$ but also a lower $T_{\text {eff }}$ near $1300 \mathrm{~K}$ could explain the overall SED for the case of $T_{\text {cr }}=1800 \mathrm{~K}$. The lower $T_{\text {eff }} \approx 1300 \mathrm{~K}$, however, could be rejected because of the strong methane bands predicted for this case ${ }^{5}$. For $T_{\text {cr }}=1700 \mathrm{~K}$, the situation is essentially the same but the methane bands are not so strong even for the case of $T_{\text {eff }} \approx 1300 \mathrm{~K}$ because of the stronger dust extinction due to the thicker cloud for the lower value of $T_{\mathrm{cr}}$, and there is no longer any reason why to reject this case ${ }^{6}$.

As another example, the overall SED of SDSS 1750 (T3.5) can be fitted with the predicted one of $T_{\text {eff }}=1100 \mathrm{~K}$ on the assumption of $T_{\text {cr }}=1800 \mathrm{~K}$ (Fig. $5 \mathrm{a}$ ), although the predicted water bands appear to be too strong (Paper II). The infrared colors of this object (Table 1), however, suggest that $T_{\text {cr }}$ should be quite high and may be as high as $T_{\text {cond }}$ (see Fig. 1b). Then, on the assumption of $T_{\text {cr }}=T_{\text {cond }}$ (case $\mathrm{C}$ or effectively no cloud), the same observed spectrum can be fitted with the predicted one of $T_{\text {eff }}=1300 \mathrm{~K}$ and the water bands show even better agreements (Fig. 5b).

\subsection{Reanalysis of the Spectral Energy Distributions}

The ambiguity due to the degeneracy of $T_{\text {eff }}$ and $T_{\text {cr }}$ in the analysis of the spectra cannot be removed if only their shapes, or relative SEDs, are analyzed as in Sect.3.1. This difficulty, however, may be relaxed to some extent if we reduce the observed spectra to the SEDs on an absolute scale. With the known distance, $d$, by the recent astrometry measurements and with the assumption that the radius, $R$, can be assumed to be the same with the Jupiter's radius, observed $\mathrm{SED}, f_{\nu}(\mathrm{Jy})$, can be transformed to an absolute scale, i.e. the emergent

\footnotetext{
${ }^{5}$ Note that the predicted spectra show bifurcations in the region of methane bands near 1.6 and $2.2 \mu \mathrm{m}$ according as the cases I (band model opacity) or II (linelist) opacities are used for $\mathrm{CH}_{4}$. As for detail, see Paper II.

${ }^{6}$ For the same reason, our previous interpretation in Paper II on 2MASS 1507 (L5) should also be reconsidered, and the low temperature model with the lower value of $T_{\text {cr }}$ should apply rather than the high temperature model with $T_{\mathrm{cr}}=1800 \mathrm{~K}$.
} 
flux from the unit surface area of the object, $F_{\nu}$, by

$$
\log F_{\nu}\left(\operatorname{erg~cm}{ }^{-2} \sec ^{-1} \mathrm{~Hz}^{-1}\right)=\log f_{\nu}(\mathrm{Jy})-2 \log (R / d)-23.4971 .
$$

Then, observed and predicted SEDs can be compared directly and no further ambiguity of vertical shift is left. We reanalyze the spectra discussed in Paper II, but we reduce all the spectra to be analyzed in this subsection to the absolute scale with the use of the parallaxes by Vrba et al. (2004) and assumption of $R=R_{\text {Jupiter }}$. The largest source of uncertainty in this transformation is the assumption on the radius, and a $30 \%$ error in the assumed radius (Burrows et al. 2001) may result in an error of $0.2-0.3 \operatorname{dex}$ on $\log F_{\nu}$.

In Fig. 6, the observed SED of the L6.5 dwarf 2MASS 1711 represented by the dots is compared with the predicted one based on the UCM with $T_{\text {cr }}=1800 \mathrm{~K}$ and $T_{\text {eff }}=1800 \mathrm{~K}$ (labeled A in the upper panel). This model was thought to fit reasonably well for this object in Paper II (see its Fig. 6). However, it now appears that the fit is very poor on the absolute scale, even though the overall shapes of the spectra agree rather well as shown in Fig. $4 \mathrm{a}$. For this object, empirical value of $T_{\text {eff }}$ is $1545 \mathrm{~K}$ and $J-K=2.25$ (Vrba et al. 2004). These data may suggest that $T_{\text {cr }} \lesssim 1700 \mathrm{~K}$ from Fig. $1 \mathrm{~b}$. We then looked for a better fit in the SEDs predicted with the UCMs of $T_{\mathrm{cr}}=1700 \mathrm{~K}$ and found that a model of $T_{\text {eff }}=1300 \mathrm{~K}(\mathrm{~B}$ in the lower panel of Fig. 6) shows a relatively good fit, except that the water bands appear to be too strong. Probably, this may not be a unique solution, but this solution is more consistent with the available observed data.

In Fig. 7, the observed SED of the L8 dwarf 2MASS1523 is compared first with the predicted one with the UCM of $T_{\text {cr }}=1800 \mathrm{~K}$ and $T_{\text {eff }}=1500 \mathrm{~K}$ (A in the upper panel). This model was deemed as the best choice for this object based on the analysis of the same spectrum without absolute calibration in Paper II (see its Fig. 8). In the upper panel of Fig. 7, the fit is very poor on the absolute scale, even though the overall shape of the spectra agree rather well. For this object, empirical value of $T_{\text {eff }}$ is $1330 \mathrm{~K}$ and $J-K=1.65$ (Vrba et al. 2004). These data may suggest that $T_{\mathrm{cr}} \approx 1700 \mathrm{~K}$ from Fig. $1 \mathrm{~b}$. We then looked for a better fit with the UCMs of $T_{\mathrm{cr}}=1700 \mathrm{~K}$ but found no reasonable solution. A model with $T_{\text {eff }}=1300 \mathrm{~K}$, (B in the lower panel of Fig. 7) shows a difference of as large as 0.2 dex on $\log F_{\nu}$ even though $T_{\text {eff }}$ is close to the empirical value. To have a better fit in the absolute scale, $T_{\text {eff }}$ may be reduced further but it will make methane bands too strong and may not be acceptable. Probably, the difference of 0.2 dex may be within the uncertainty of the absolute flux due to our assumption on the radius as noted above, and we suggest that a possible solution can be $T_{\mathrm{cr}} \approx 1700 \mathrm{~K}$ and $T_{\text {eff }} \approx 1300 \mathrm{~K}$.

In Fig. 8, we show the observed SED of T2 dwarf SDSS 1254 by the dots and compared it with the predicted one based on the UCM of $T_{\text {cr }}=1800 \mathrm{~K}$ and $T_{\text {eff }}=1300 \mathrm{~K}$ (A in the upper panel). This is the model shown in Fig. 9 of Paper II and suggested to be the best 
model based on the fit of the overall shapes of the observed and predicted spectra. It is now confirmed that the fit is also fine in the absolute scale. Also, $J-K=0.82$ (Knapp et al. 2004 ) of this object may suggest that $T_{\mathrm{cr}} \approx 1800 \mathrm{~K}$ from Fig. $1 \mathrm{~b}$. The value of $T_{\text {eff }}$ is only slightly lower than the empirical value of $T_{\text {eff }}=1361 K$ (Vrba et al. 2004). We examined the case of $T_{\text {eff }}=1400 \mathrm{~K}$ which required $T_{\text {cr }}=1900 \mathrm{~K}$ to reproduce the overall shape of the observed spectrum. This case (B in the lower panel of Fig. 8) shows some deviation from the observed spectrum, although the deviation may be within the uncertainty of the absolute scale as noted above.

Finally, we examine the case of T3.5 dwarf SDSS 1750 in Fig. 9, in which the observed SED is compared with the predicted ones from the two different UCMs. First, the case of $T_{\text {cr }}=1800 \mathrm{~K}$ and $T_{\text {eff }}=1100 \mathrm{~K}$, which we have accepted in Paper II, reproduces the relative SED reasonably well except that water bands appear to be too strong, and we have further suggested $\log g=5.5$ to reduce the water band strengths in Paper II (see its Fig. 10). The fit in the absolute scale for this model is not so good in Fig. 9 even though it may be within the uncertainty of the absolute flux (A in the upper panel). For comparison, recent empirical value is $T_{\text {eff }}=1478 \mathrm{~K}$ (Vrba et al. 2004), about $400 \mathrm{~K}$ higher than the value based on the model fitting. Also, this object is as blue as $J-K=0.12$ (Knapp et al. 2004) and the dust cloud may have cleared already at T3.5 as can be inferred from Fig. 1b. We then apply our model with $T_{\text {cr }}=T_{\text {cond }}$ (case $\mathrm{C}$ ) and looked for the best fit. We found that a model of $T_{\text {eff }}=1300 \mathrm{~K}$ provides the best fit both in relative and absolute scales (B in the lower panel of Fig. 9). Another photometry suggests that $J-K=0.83$ (Vrba et al. 2004) and we also examined the case of $T_{\text {cr }}=1900 \mathrm{~K}$. It turns out that the lower values of $T_{\text {cr }}$ and/or the higher values of $T_{\text {eff }}$ do not improve the fit in relative as well as in absolute scale.

The possible best values of $T_{\text {eff }}$ based on the reanalysis outlined above are summarized in Table 1 together with some observed data, and will be discussed in Sect.3.3.

\subsection{Interpretation of the L-T Spectral Sequence}

As an example of the L-T spectral sequence, we reproduce in Fig. 10 the spectra of cool dwarfs from L6.5 to T3.5, which were discussed in some detail elsewhere (Nakajima, Tsuji, \& Yanagisawa 2004). The spectra show systematic changes from the $\mathrm{L}$ dwarfs with $\mathrm{H}_{2} \mathrm{O}$ bands of modest strengths and marginal $\mathrm{CH}_{4}$ bands to the $\mathrm{T}$ dwarfs with strong bands of both $\mathrm{H}_{2} \mathrm{O}$ and $\mathrm{CH}_{4}$. Also, the overall SEDs in the spectral range of Fig. 10 change from red in the $\mathrm{L}$ dwarfs to blue in the $\mathrm{T}$ dwarfs. This spectral sequence could have been interpreted as a temperature sequence which extends from 1800 to $1100 \mathrm{~K}$ ( reproduced in the 6 -th column of Table 1), so long as we assume a uniform value of $T_{\text {cr }}=1800 \mathrm{~K}$ throughout $\mathrm{L}$ and $\mathrm{T}$ dwarfs 
(Paper II). This seemed to be a reasonable result for such a large and systematic change of the spectra, and also fitted to the general expectation that the L-T spectral classification may be an extension of the stellar spectral types beyond $\mathrm{M}$ to the cooler temperatures.

In Sect.3.2, we reanalyzed the same data reproduced in Fig. 10 with the $T_{\text {cr }}$ values inferred from the $J-K$ values of individual objects (Table 1 and Fig. 1b). Also fitting of the observed and predicted spectra is done not only by the relative shape of the spectra but also by the use of the absolute flux scale based on the recent astrometry data. The result of the reanalysis turns out to be quite different as summarized in the 7-th column of Table 1. The spectra from L6.5 to T3.5 shown in Fig. 10 can be interpreted by a uniform value of $T_{\text {eff }} \approx 1300 \mathrm{~K}$ throughout. Instead, the spectral sequence of Fig. 10 can be explained by the change of $T_{\text {cr }}$ from about $1700 \mathrm{~K}$ to $T_{\text {cond }}$ (i.e. case C) ${ }^{7}$. An important conclusion is that the effect of $T_{\mathrm{cr}}$ is sometimes more important than that of $T_{\text {eff }}$, and such drastic change of spectra that requires different spectral types $\mathrm{L}$ and $\mathrm{T}$ can be due to the change of $T_{\text {cr }}$ rather than of $T_{\text {eff }}$.

Thus, the present reanalysis shows a marked contrast to the previous analysis (Paper II), and it is quite unexpected that the L-T spectral sequence can no longer be regarded as a temperature sequence, but represents a sequence of the change of the dust column density in the observable photosphere at least between the middle L and early T dwarfs. Nevertheless, we should accept the new result which is based on the analysis of the SEDs on an absolute scale. Even though the absolute flux may be uncertain by as much as $\Delta \log F_{\nu} \approx 0.2-0.3$ dex because of the uncertainty of the radius of an individual object as noted in Sect.3.2, the ambiguity in the analysis of the relative SED should be still larger as shown in Sect.3.2. Also, the critical temperature $T_{\text {cr }}$ is already selected to be consistent with the infrared colors (Fig. 1b), and the resulting $T_{\text {eff }}$ values agree with the recent empirical effective temperatures which suggested an almost uniform value of $T_{\text {eff }} \approx 1400 \mathrm{~K}$ in the same spectral range (Vrba et al. 2004; Golimowski et al. 2004). A systematic difference of $100 \mathrm{~K}$ remains to be explained, but such a difference may be within the error bars of the both analyses.

\section{BASIC PHYSICAL PROPERTIES OF ULTRACOOL DWARFS}

Recent progress in observations of such faint objects as $\mathrm{L}$ and $\mathrm{T}$ dwarfs is quite substantial, especially in photometry (e.g. Leggett et al. 2002; Golimowski et al. 2004; Knapp

\footnotetext{
${ }^{7}$ In this interpretation, L6.5 dwarf 2MASS 1711 and L8 dwarf 2MASS 1523 have the same $T_{\text {eff }}$ and the same $T_{\text {eff }}$ (Figs. $6 \& 7$ ), but we are now discussing the first order effect with models in which $T_{\text {eff }}$ and $T_{\text {cr }}$ are changed by a step of $100 \mathrm{~K}$, and a further fine tuning should certainly be needed.
} 
et al. 2004) and astrometry (e.g. Dahn et al. 2002; Tinney, Burgasser \& Kirkpatrick 2003; Vrba et al. 2004). Thus, we now have reasonably accurate empirical data on luminosities and effective temperatures for a large sample of $\mathrm{L}$ and $\mathrm{T}$ dwarfs. At the same time, these new data uncovered a difficulty in the spectral classification of $\mathrm{L}$ and $\mathrm{T}$ dwarfs, which was essentially following the methodology of the stellar spectral classification.

\subsection{Luminosities}

Recent progress in observations finally provided reasonably accurate absolute magnitudes (monochromatic as well as bolometric) for a large sample of L and T dwarfs (e.g. Dahn et al. 2002; Tinney et al. 2003; Vrba et al. 2004). As an example, we reproduce the $M_{J}$ plotted against the L-T spectral type in Fig. 11a with the data by Vrba et al. (2004) (see their original plot as for more detail with error bars), showing the so-called $J$-brightening at the early $\mathrm{T}$ dwarfs. It is known that the situation is more or less the same for $M_{H}$ and $M_{K}$ if not so pronounced as in $M_{J}$ (e.g. Dahn et al. 2002; Tinney et al. 2003; Vrba et al. 2004). Then, these data may suggest that the bolometric magnitude $M_{\text {bol }}$ should also show the similar brightening, since most of the flux is emitted in $J, H$, and $K$ bands. We confirmed in Fig. 11b that the absolute bolometric magnitude $M_{\text {bol }}$ plotted against the L-T spectral type with the data from Vrba et al. (2004) in fact shows the brightening at the early $\mathrm{T}$ dwarfs. The values of bolometric magnitude $M_{\text {bol }}$ used in Fig. $11 \mathrm{~b}$ are already based on the bolometric corrections by Golimowski et al. (2004) including the result of the $L^{\prime}$ and $M^{\prime}$ photometry. However, we examine the $M_{\text {bol }}$ values of Golimowski et al. (2004) based on a different photometric system in Fig. 11c. The "brightening" may not be so clear as in Fig. 11b, but the $M_{\text {bol }}$ also levels off between L5 and T4 in Fig. 11c.

Although these results are quite unexpected, it is more easy to interpret Fig. 11b (or Fig. 11c) than Fig. 11a: Since the bolometric luminosities essentially reflect the cooling of brown dwarfs, the brightening (or the level-off) of the total luminosities is difficult to understand if they are plotted against a correct temperature indicator. An only possible solution may be that the problem is not in the ordinate but in the abscissa of Fig.11b (or Fig. 11c), namely the L-T spectral sequence is not representing a temperature sequence. Since Fig. 11a and Fig. 11b are based on the same photometry data and show quite similar "brightening", the brightening in Fig. 11a should most probably be due to the same origin as in Fig. 11b. This result implies that the so-called " $J$ - brightening" is not due to any atmospheric effect, but should be due to intrinsic properties of the early $\mathrm{T}$ dwarfs. This result that the L-T spectral sequence is not a temperature sequence is consistent with the recent empirical effective temperatures (e.g. Golimowski et al. 2004; Vrba et al. 2004) and with the conclusion 
of Sect.3.3 based on the analysis of a limited sample of $\mathrm{L}$ and $\mathrm{T}$ dwarfs. All these results in turn lend a support to our interpretation on the "brightening" . Thus, the so-called "J-brightening" problem on the $M_{J}$ - Sp. Type plot no longer exists, while that on the CM diagram has been solved already (Sect.2.3).

\subsection{Effective Temperatures}

Effective temperature is one of the basic fundamental parameters that specify the nature of substellar as well as of stellar object. Accurate determination of $T_{\text {eff }}$, however, is generally difficult because the total energy flux integrated over the whole spectral region is required on an absolute scale (i.e. in unit of energy flux emerging from the unit surface area of the object). In the case of star, observed bolometric flux can be converted to the absolute emergent total flux if the stellar angular diameter could be measured. In the case of brown dwarf, the angular diameter is more difficult to measure at present but its equivalence can be estimated if the distance can be known, because of the favorable nature of brown dwarfs that the radius is within $30 \%$ of the Jupiter's radius independently of mass (e.g. Burrows et al. 2001).

Recently, reasonably accurate trigonometric parallaxes are made available thanks to the elaborate astrometric observations by several groups (e.g. Dahn et al. 2002; Tinney et

al. 2003; Vrba et al. 2004). These results are combined with the photometric observations well extended to the $L^{\prime}$ and $M^{\prime}$ regions (e.g. Leggett et al. 2002; Golimowski et al. 2004; Knapp et al. 2004) and empirical effective temperatures as well as the absolute magnitudes are determined for dozens of $\mathrm{L}$ and $\mathrm{T}$ dwarfs. An important finding is that the empirical $T_{\text {eff }}$ 's by Vrba et al. (2004) and by Golimowski et al. (2004) show a large plateau between about L5 and T5 and, especially, $T_{\text {eff }}$ between L 8 and T2 is nearly constant. This result was also suggested by Nakajima et al. (2004) based on the bolometric correction predicted with the use of the UCMs. The effect of $\log g$ may not be so large as to modify the qualitative nature of the spectra. Thus the $\mathrm{L} / \mathrm{T}$ transition as well as the change of the spectra between late $\mathrm{L}$ and early $\mathrm{T}$ types should not be due to $T_{\text {eff }}$ and $\log g$ alone but may be governed by another parameter, which we have already identified with $T_{\text {cr }}$.

With the empirical $T_{\text {eff }}$ as the abscissa, we plot $M_{J}$ in Fig. 12a. Since $T_{\text {eff }}$ is derived from $M_{\text {bol }}$ which is not fully independent of $M_{J}$, it is expected from the beginning that $M_{J}$ and $T_{\text {eff }}$ are somewhat related. Nevertheless, if the $J$-band flux suffers a serious atmospheric effect, such a smooth monotonic curve as Fig. 12a may not necessarily be trivial. It is confirmed that the "brightening" such as shown in Fig. 11a disappears if $M_{J}$ is plotted against effective temperature, and this fact can be regarded as a confirmation of the conclusion of Sect.4.1 
that the so-called " $J$-brightening" is a problem of L-T classification rather than of $M_{J}$. Also, it is to be noted that the scattering is rather small in Fig. 12a except for the $\mathrm{L} / \mathrm{T}$ transition region. We overlaid in Fig. 12a the evolutionary tracks of 10,42 , and $70 M_{\text {Jupiter }}$ transformed to $M_{J}-T_{\text {eff }}$ from $M_{\text {bol }}-T_{\text {eff }}$ (Burrows et al. 1997) for different values of $T_{\mathrm{cr}}$. It can be confirmed that the variable $T_{\text {cr }}$ in fact results in the scatters in $M_{J}$ at $T_{\text {eff }} \approx 1500 \mathrm{~K}$ where the effect of the dust clouds is the largest, but that the "atmospheric effect" is not so large as to produce the so-called "J-brightening".

To investigate the nature of the empirical $T_{\text {eff }}$, we also plot $M_{\text {bol }}$ against the empirical $T_{\text {eff }}$ (error bars in the original data are not shown, but typically $\pm 200 \mathrm{~K}$ ) in Fig. $12 \mathrm{~b}$, and the evolutionary tracks for 10,42 , and $70 M_{\text {Jupiter }}$ (Burrows et al. 1997) are overlaid. The empirical values of $T_{\text {eff }}$ are derived with the assumption of the uniform radius of $0.90 R_{\text {Jupiter }}$ by Vrba et al. (2004) with their eqn.(7), and the results seem to be approximately equivalent to have derived $T_{\text {eff }}$ with the use of the evolutionary tracks for $42-70 M_{\text {Jupiter }}$. Thus, the empirical $T_{\text {eff }}$ 's are subject to some ambiguities due to the uncertainties of mass, radius, and/or age. Other authors (e.g. Golimowski et al. 2004; Knapp et al. 2004) applied the evolutionary tracks to convert $M_{\text {bol }}$ to $T_{\text {eff }}$, but the exact evolutionary status of an individual object is anyhow unknown. For example, Golimowski et al. (2004) showed that the range of possible $T_{\text {eff }}$ derived from $L_{\text {bol }}$ assuming evolutionary models with ages of 0.1 - $10 \mathrm{Gyr}$ is $\approx 300 \mathrm{~K}$. In Fig. $12 \mathrm{c}$, we also plot $M_{\text {bol }}$ against the empirical $T_{\text {eff }}$ derived for an age of $\approx 3$ Gyr by Golimowski et al. (2004). Except for a few cases, Fig. 12b and Fig. 12c appear to be rather similar, and it looks as if most brown dwarfs are on the evolutionary tracks of 42-70 $M_{\text {Jupiter. }}$ However, this is simply a consequence that the age of most brown dwarfs are assumed to be $\approx 3 \mathrm{Gyr}$.

More or less similar difficulty has been noticed by Golimowski et al. (2004), who compared the diagrams of $M_{K}, M_{L^{\prime}}$ and $M_{M^{\prime}}$ versus $T_{\text {eff }}$ with the predictions based on the models of Marley et al. (2002) for varying sedimentation efficiencies $f_{\text {sed }}(3,5$, and no cloud) and different values of $\log g(4.5,5.0$, and 5.5). Their results showed that the model predictions reproduce well the broad ranges of observed absolute magnitudes and effective temperatures for a large sample of $\mathrm{L}$ and $\mathrm{T}$ dwarfs. Yet it appeared to be difficulty to decide particular sets of model parameters because of the difficulty of knowing masses, ages, and metallicities of individual objects. Golimowski et al. (2004) also showed that the L3 - T4.5 dwarfs generally have higher gravities than the T6 - T9 dwarfs, and these results may be consistent with our Fig. 12 in that earlier dwarfs generally tend to populate the evolutionary tracks of the higher mass models.

Despite the difficulty of observing such faint objects as brown dwarfs, one relief is that parallaxes could have been measured accurately. In fact, the recent achievement of 
measuring the parallaxes of dozens of ultracool dwarfs is the most important contribution to our understanding of brown dwarfs (e.g. see Sect.2.1 and Sect.4.1). Once distance of object can be known accurately, it could be expected that we can understand well the nature of astronomical object. Nevertheless, it appeared that the empirical $T_{\text {eff }}$ values for individual objects are still subject to uncertainty of about $\pm 200 \mathrm{~K}$ (Vrba et al. 2004; Golimowski et al. 2004; Knapp et al. 2004) and, for this reason, it is still difficult to determine masses and/or ages of individual objects from the observed data at present. We must wait for direct measurements of radii or angular diameters for a final solution of this uncertainty. As a compromise, model analyses such as given in Sect.3 can be of some use to examine the consistency of various observed data, and to improve our knowledge on $T_{\text {eff }}$ and other parameters.

\subsection{Spectral Classification}

A conclusion to be drawn from Sects 3.3 and 4.1 is that the L-T spectral classification may have serious problem regarding its meaning as a temperature classification. The stellar spectral classification has been done on purely empirical basis and it was not intended to be a temperature sequence from the beginning. However, the great success of the stellar spectral classification established at the beginning of the 20-th century is largely due to the finding that the spectral sequence is a temperature sequence. Later efforts to interpret the stellar spectral sequence in terms of temperature and other physical parameters finally resulted in establishing the present-day stellar astrophysics. The present L - T spectral classification has also been done on purely empirical basis (e.g. Kirkpatrick et al. 1999) and again it was not explicitly mentioned that the resulting spectral sequence will be a temperature sequence. Thus it is left free as to how to interpret the $\mathrm{L}-\mathrm{T}$ spectral types. However, the $\mathrm{L}$ and $\mathrm{T}$ types are conceived as extensions of the spectral type beyond $\mathrm{M}$, and may implicitly be expected to be temperature indicators.

Anyhow, we should first understand what the present L - T spectral types mean. Although the reason for the difficulty to interpret the $\mathrm{L}$ - T spectral types can be explained as due to the degeneracy of $T_{\text {eff }}$ and $T_{\text {cr }}$ on the spectra contaminated with dust (Sect.3.1), at least partly, this problem may be most serious in the region around $T_{\text {eff }} \approx 1400 \pm 100$ where the transition from $\mathrm{L}$ to $\mathrm{T}$ takes place (Fig. 1). In other regions, however, the situation may be different, and the L - T spectral classification may be used as a temperature classification if applied with some cautions. We now apply our UCM as a working model to interpret the L-T classification in three different parts, namely, early L dwarfs (roughly $T_{\text {eff }}>1500 \mathrm{~K}$ ),

L- $\mathrm{T}$ transition region $\left(1300 \mathrm{~K} \lesssim T_{\text {eff }} \lesssim 1500 \mathrm{~K}\right)$, and dust-cleared $\mathrm{T}$ dwarfs $\left(T_{\text {eff }}<1300 \mathrm{~K}\right)$ : 
In the early $\mathrm{L}$ dwarfs, the dust clouds that occupy the region of $T_{\text {cr }} \lesssim T \lesssim T_{\text {cond }}$ are entirely located in the optically thin region. In this case, the location of the lower boundary of the cloud at $T \approx T_{\text {cond }}$ should be more important than that of the upper boundary at $T \approx T_{\text {cr }}$, since the region near the upper boundary of the dust cloud will have little contribution to the dust column density because of the lower density there. For this reason, the spectra essentially depend on $T_{\text {cond }}$ which in turn can be well interpreted in terms of $T_{\text {eff }}$ and $\log g$, and this explains why scatters are small in the early L dwarfs in Fig. 1. At the same time, it is difficult to known the value of $T_{\text {cr }}$ from the infrared colors (Fig. 1b).

From middle $\mathrm{L}$ to early $\mathrm{T}$ dwarfs, the $\mathrm{L}-\mathrm{T}$ spectral classification met serious difficulty as noted above. In this region, the lower boundary of the cloud at $T \approx T_{\text {cond }}$ will be in the unobservable photosphere (i.e. in $\tau>1$ ), and the thickness of the cloud in the observable photosphere is essentially determined by $T_{\mathrm{cr}}$, which is not controlled by $T_{\text {eff }}$ nor by $\log g$. For this reason, the spectra show little effect of $T_{\text {eff }}$ but depend mostly on $T_{\text {cr }}$, and an actual example of this case is shown by the spectral sequence reproduced in Fig. 10. The analysis outlined in Sect.3.2 is based on a limited sample which, however, may represent the general feature of the spectra between middle $\mathrm{M}$ and early $\mathrm{T}$ types. This is because a larger sample shows the same conclusion: First, empirical $T_{\text {eff }}$ plotted against spectral types shows a large plateau in the middle part of the L-T sequence (Vrba et al. 2004; Golimowski et al. 2004; Nakajima et al. 2004; Leggett et al. 2005). Second, $M_{\text {bol }}$ plotted against spectral types shows a "brightening" around early T types (Fig. 12b), which is difficult to understand if the spectral types are on a correct temperature sequence. Thus the spectral classification between middle $\mathrm{L}$ and early $\mathrm{T}$ types may radically be reconsidered.

In the middle and late $\mathrm{T}$ dwarfs, the effect of cloud is diminishing because of the immersion of the cloud in the optically thick region, and gaseous opacities dominate. The upper boundary of the cloud may still in the observable photosphere in the middle $\mathrm{T}$, but it seems that $T_{\text {cr }}$ may be systematically high in these dwarfs (about $1900 \mathrm{~K}$ or higher from Fig. $1 \mathrm{~b}$ and Fig. 2b). This fact may suggest a possibility that the dust cloud is already quite thin in middle $\mathrm{T}$ dwarfs, but the reason for this is unknown. Finally, in the late $\mathrm{T}$ dwarfs, dust cloud is in the optically thick region and no information on the cloud can be obtained from observations (Liebert et al. 2000). In this case, $T_{\text {cond }}$ as well as $T_{\text {cr }}$ has no effect on spectra as well as on colors. The scatters in colors at the fixed $T_{\text {eff }}$ are mostly due to gravity effect as noted in Sect.2.

Apart from the problems related to $T_{\mathrm{cr}}$, one difficulty in the classification of dusty dwarfs is that the dust itself shows no clear spectral signature and it is very difficult to estimate the dust column density directly from observations. The spectral classification had to be done with the use of the spectral features originating from atoms and molecules, while the 
spectra are largely controlled by dust. For example, methane bands which are used in the infrared classification show drastic change from L to T dwarfs (e.g. Fig. 10) but this is not due to a direct effect of the change of the methane abundance with $T_{\text {eff }}$ but rather due to an indirect effect of the change of the dust extinction with $T_{\mathrm{cr}}$ as discussed in Sect.3.3. But there should be a case that the methane bands actually change due to the change of the methane abundance with $T_{\text {eff }}$. For this reason, methane bands cannot be indicators of temperature, and the situation may be more or less the same for other atomic and molecular features.

Another difficulty of the present L - T classification has also been pointed out in that the L types based on the optical spectra and those on the near infrared spectra show differences as large as 3 subtypes (e.g. Stephens 2003; Knapp et al. 2004; Leggett et al. 2005). This fact, however, may offer an interesting possibility of the two-dimensional spectral classification of ultracool dwarfs, if the optical types can be indicators of temperature and the infrared types of the dust opacities (Stephens 2003). In view of the difficulties outlined above, however, it seems to be more difficult to separate the effects of temperature and of dust opacities. For example, the effect of the dust column density is more serious as to produce such a large difference in spectral types from L6.5 to T3.5 for about the same $T_{\text {eff }}$ as shown in Fig. 10 rather than results in just 3 subclasses at the largest in late $\mathrm{L}$ dwarfs.

The present L-T classification is essentially following the methodology of the stellar spectral classification (e.g. Kirkpatrick et al. 1999), which is a marvelous art to infer the basic stellar properties such as the effective temperature and luminosity by just looking at the low resolution spectra. In the case of the spectra of cool dwarfs contaminated with dust, such a convention may be more difficult not only because an additional parameter related to the dust clouds is required to characterize the spectra but also the dust itself shows no spectral signature. As a parameter to specify the property of the dust cloud, we introduced $T_{\text {cr }}$, which is a measure of the dust column density in our UCM, but the real difficulty is that the effects of $T_{\text {eff }}$ and $T_{\text {cr }}$ cannot be separated even if the effect of $\log g$ could be known. It is of course desirable to be able to know the basic parameters such as $T_{\text {eff }}, \log g$, and metallicity, and further some dust properties (e.g. $T_{\text {cr }}$ in our UCMs) by just looking at the low resolution spectra. However, it seems to be a formidable problem to have a classification scheme similar to the stellar spectral classification for the spectra of dusty dwarfs, and more detailed and careful examination of the spectra from optical to infrared region should be required. 


\section{DISCUSSION AND CONCLUDING REMARKS}

Recent observations of ultracool dwarfs revealed some confusion as to their interpreta-

tion. For example, the "brightening" in the infrared absolute magnitudes at the early $\mathrm{T}$ dwarfs on the L-T sequence as well as on the CM diagrams (e.g. Dahn et al. 2002; Tinney et al. 2003; Vrba et al. 2004), large scatters in the infrared colors (Knapp et al, 2004 and references cited therein), a large plateau in $T_{\text {eff }}$ - Sp. Type calibration between middle L and middle T (Vrba et al. 2004) etc. We have shown in this paper that these difficulties are mostly resolved if we apply our UCMs properly, i.e., if we allow the critical temperature to change from as high as the condensation temperature to near the surface temperature. We have not yet considered another curious observation that the absorption bands of FeH decrease first from early to late $\mathrm{L}$ types but strengthen again at early $\mathrm{T}$ types (Burgasser et al. 2002b; Nakajima et al. 2004). We have no enough data to analyze this problem in detail, but we suggest that this may be the same phenomenon as the "J-brightening" at the early $\mathrm{T}$ types. In other words, if the FeH band strength is plotted against $T_{\text {eff }}$ rather than L-T types, it will show a steady decrease and we hope that this will be confirmed by the future observations. Also, if this is confirmed, FeH can be a useful marker for spectral classification.

Within the framework of UCM, the critical temperature $T_{\text {cr }}$ shows such a large variation at a given $T_{\text {eff }}$, and this result implies that the dust column density in the observable photosphere differs largely for the same $T_{\text {eff }}$ (and other basic parameters). It is remarkable that the variation of $T_{\text {cr }}$ is especially large at $T_{\text {eff }} \approx 1400 \pm 100 \mathrm{~K}$, and $\mathrm{T}$ dwarfs in this region mostly show $T_{\text {cr }} \gtrsim 1800 \mathrm{~K}$ while $\mathrm{L}$ dwarfs $T_{\text {cr }}<1800 \mathrm{~K}$ (Fig. 1b). Then $T_{\text {cr }}$ is as high as $1900 \mathrm{~K}$ or near $T_{\text {cond }}$ in middle $\mathrm{T}$ dwarfs as noted in Sects.2.2 and 4.3. These results may indicate that the thickness of the dust cloud itself is decreasing after the L-T transition and further throughout $\mathrm{T}$ dwarfs. Thus it appears that thinning of the dust cloud should be associated with the immersion of the cloud in $\mathrm{T}$ dwarfs, but it is not clear if this is also the case in $\mathrm{L}$ dwarfs. A problem is if the dust cloud will finally disappear in late $\mathrm{T}$ dwarfs or if it still exists in the deeper layer. Unfortunately, this problem cannot be answered with observations, since no information is available from the optically thick region.

It is to be remembered that the immersion of the dust cloud in cooler dwarf is a natural consequence of the change of the photospheric structure according as $\mathrm{L}$ dwarf evolve to $\mathrm{T}$ dwarf, and this is simply because the dust cloud always forms at about the same temperature, namely, at $T_{\text {cond }} \approx 2000 \mathrm{~K}$, where is in the optically thin (thick) region of the objects with relatively high (low) $T_{\text {eff }}$. However, the thinning of the dust cloud is more difficult to understand by such a simple picture. A known noticeable change of the photospheric structure at $T_{\text {eff }} \approx 1400 \mathrm{~K}$ is the formation of the second (outer) convective zone (Paper I), which may have some impact on the formation and destruction of the clouds. Since the convective 
activities may depend on the age and evolutionary history of the cooling brown dwarfs, the nature of cloud including $T_{\text {cr }}$ may depend on such effects as well. However, the details of how the convective activity is related to the variation of $T_{\mathrm{cr}}$ is unknown at present.

The critical temperature $T_{\text {cr }}$ and its variation should be a key in our understanding of the photospheric structure and hence of the observed properties of dusty dwarfs. In this paper, however, we leave $T_{\text {cr }}$ as a free parameter to be estimated from observations. It is of course desirable that $T_{\text {cr }}$, or more generally the upper boundary of the dust cloud, can be determined from the basic physics. However, this will anyhow be highly model dependent at present. For example, Woitke \& Helling (2004) recently proposed an interesting model in which a series of processes such as nucleation, dust growth, gravitational settling, evaporation, and element replenishment will take place in a convective life cycle in the photosphere, and the structure of the dust cloud including its upper boundary as well as dust properties such as the grain size can be determined by solving their moment equations in the circulating flow. In this model, however, everything depends on a parameter referred to as the mixing timescale, which was assumed as a measure of the efficiency of the convective activity including overshooting. But it is by no mean clear if the mixing will take place in such a way as to interact with the dust formation so nicely as assumed in their model. For example, the structural models of cool dwarfs show that convective zone is situated deeper in L dwarfs than in T dwarfs (e.g. Tsuji 2002), and hence mixing in the photosphere induced by the convection will be more effective in $\mathrm{T}$ dwarfs than in $\mathrm{L}$ dwarfs. Then, if the mixing plays a major role in cloud formation, a question is why $\mathrm{T}$ dwarfs are not dusty while $\mathrm{L}$ dwarfs are.

Our major concern at present is to interpret observed data in terms of the basic stellar parameters such as $T_{\text {eff }}, \log g$, metallicity, chemical composition etc. For this purpose, it is required to predict observables such as colors, magnitudes, spectra etc. and our empirical model referred to as UCM is primarily developed for such applications in mind. So far as the model is empirical, the cloud property such as $T_{\text {cr }}$ (i.e. the upper boundary of the cloud) is simply estimated empirically and hence free from any particular model of cloud formation, which is presently not well established yet. Such an empirical approach still plays an important role in our studies of stellar and substellar photospheres. For example, stellar convection theories, which we have also employed in our UCMs, mostly involve a parameter known as the mixing length, and the micro-turbulent velocity is usually determined empirically rather than evaluated theoretically for individual object. Of course an empirical approach is only an initial step toward more complete modeling, but we must recognize that the stellar and substellar photospheres still involve formidable problems for which fully complete solution is difficult at present.

Finally, it is to be noted that the critical temperature $T_{\text {cr }}$ is a parameter inherent to 
our UCMs, and the fifth parameter next to the $T_{\text {eff }}, \log g$, chemical composition, and microturbulent velocity may not necessarily be restricted to $T_{\mathrm{cr}}$. For example, other parameter that characterizes the dust clouds, such as the sedimentation efficiency $f_{\text {sed }}$ in the models of Marley et al. (2002), will play similar role as our $T_{\mathrm{cr}}$, and their cloudy models have extensively been applied to the interpretation and analyses of the recent observations of $\mathrm{L}$ and T dwarfs (e.g. Knapp et al. 2004; Golimowski et al. 2004). From these analyses and from ours outlined in this paper, the cloudy models of Marley et al. (2002) and our UCMs basically agree in that the cloud properties depend on a dynamical process whose origin cannot be identified yet and whose effect had to be represented by a variable parameter such as $f_{\text {sed }}$ or $T_{\text {cr }}$. In practical applications, our $T_{\text {cr }}$ may have some advantages in that it can easily be incorporated in the classical non-grey theory. Also, $T_{\text {cr }}$ has a clear physical meaning related to the thickness of the dust clouds, and hence can be inferred directly from the observed infrared colors. Furthermore, we have shown that almost all the data points in the large collective data such as the two-color diagram and CM diagram can be consistently interpreted with the $T_{\mathrm{cr}}$ values in the appropriate range, and this fact implies that $T_{\mathrm{cr}}$ is a reasonable choice as a parameter for the characterization of dusty dwarfs.

In conclusion, we believe that we have now applied our UCMs ${ }^{8}$ more properly to the interpretation and analysis of the observed data of dusty dwarfs, with the recognition that the parameter $T_{\text {cr }}$ takes different values in addition to $T_{\text {eff }}$ and $\log g$ (under the fixed chemical composition and micro-turbulent velocity). We certainly realized that dust plays an important role in determining the observed properties of cool dwarfs at an early time, but we did not fully understand its effect nor treated it properly until now. We should now recognize that the dust is one of the fundamental elements in the characterization of dusty dwarfs. Based on this recognition, we must reexamine the observed data in more detail and reconsider such a basic problem as the spectral classification of ultracool dwarfs, which cannot be a simple extension of the stellar spectral classification. A relatively short history of the studies on ultracool dwarfs including brown dwarfs was already quite intriguing, and thus such ultracool dwarfs will remain to be exciting subjects of further observational and theoretical challenges.

I thank Tadashi Nakajima for useful discussion and helpful comments on the draft of this paper, and Mark Marley (as the referee) for constructive criticism and invaluable suggestions which helped to improve the initial version of this paper. This work was supported by the grant-in-aid No.15204010 of JSPS (PI: M. Fujimoto) and carried out with the use of the

\footnotetext{
${ }^{8} \mathrm{As}$ for an extended and updated version of the UCMs, see our web site at http://www.mtk.ioa.s.utokyo.ac.jp/ ${ }^{\sim}$ ttsuji/export/ucm
} 
facilities at the Astromonical Data Analysis Center of NAO, which is an interuniversity research institute of astronomy operated by the Ministry of Education and Science.

\section{REFERENCES}

Ackerman, A. S. \& Marley, M. S. 2001, ApJ, 556, 872

Becklin, E. E., \& Zuckermann, B. A. 1988, Nature, 336, 656

Burgasser, A. J., et al. 2002a, ApJ, 564, 421

Burgasser, A. J., Marley, M. S., Ackerman, A. S., Saumon, D., Lodders, K., Dahn, C. C., Harris, H. C., \& Kirkpatrick, J. D. 2002b, ApJ, 571, L151

Burrows, A., et al. 1997, ApJ, 491, 856

Burrows, A., Hubbard, W. B., Lunine, J. I., \& Liebert, J. 2001, Rev. Mod. Phys., 73, 719

Chabrier, G., Baraffe, I., Allard, F., \& Hauschildt, P. 2000, ApJ, 542, 464

Chandrasekhar, S. 1939, An Introduction to the Study of Stellar Structure (Chicago: Univ. Chicago Press)

Dahn, C. C., et al. 2002, AJ, 124, 1170

Geballe, T. R., et al. 2002, ApJ, 564, 466

Golimowski, D. A., et al. 2004, AJ, 127, 3516

Kirkpatrick, J. D., et. al. 1999, ApJ, 519, 802

Knapp., G. R., et al. 2004, AJ, 127, 3553

Leggett, S. K., et al. 2002, ApJ, 564, 452

Leggett, S. K., Allard, F., Burgasser, A. J., Jones, H. R. A., Marley, M. S., \& Tsuji, T. 2005, Proc. 13-th Cool Stars Workshop, eds. F. Favata et al., EAS SP, in press (astro$\mathrm{ph} / 0409389)$

Liebert, J., Reid, I. N., Burrows, A., Burgasser, A. J., Kirlpatrick, J. D., \& Gizis, J. E. 2000, ApJ, 533, L155

Marley, M. S., Seager, S., Saumon, D., Lodders, K., Ackerman, A. S., Freedman, R., \& Fan, X. 2002, ApJ, 568, 335 
Marley, M. S., Cushing, M. C., \& Saumon, D. 2005, Proc. 13-th Cool Stars Workshop, eds. F. Favata et al., EAS SP, in press (astro-ph/0409267)

Martín, E. L., Delfosse, X., Basri, G., Goldman, B., Forveille, T., \& Zapatero Osorio, M. R. 1999, AJ, 118, 2466

Nakajima, T., Oppenheimer, B. R., Kulkarni, S. R., Golimowski, D. A., Matthews, K., Durrance, S. T. 1995, Nature, 378, 463

Nakajima, T., Tsuji, T., \& Yanagisawa, K. 2004, ApJ, 607, 499

Reid, I. N., Burgasser, A. J., Cruz, K. L., Kirkpatrick, J. D., \& Gizis, J. E. 2001, AJ, 121, 1710

Stephens, D. C. 2003, in IAU Symp. 211, Brown Dwarfs, ed. E. Martín (San Francisco: ASP), 355

Struve, O., \& Elvey, C. T. 1934, ApJ, 79, 409

Testi, L., et al. 2001, ApJ, 552, L147

Tinney, C. G., Burgasser, A. J., \& Kirkpatrick, J. D. 2003, AJ, 126, 975

Tokunaga, A. T., Simon, D. A., \& Vacca, W. D. 2002, PASP, 114, 180

Tsuji, T. 2001, in Ultracool Dwarfs - New Spectral Types L and T, ed. H. R. A. Jones \& I. A. Steele (Berlin: Springer-Verlag), 9

Tsuji, T. 2002, ApJ, 575, 264 (Paper I)

Tsuji, T. 2005, Proc. 13-th Cool Stars Workshop, eds. F. Favata et al., EAS SP, in press (astro-ph/0410067)

Tsuji, T., \& Nakajima, T. 2003, ApJ, 585, L151

Tsuji, T., Nakajima, T., \& Yanagisawa, K. 2004, ApJ, 607, 511 (Paper II)

Vrba, F. J., et al. 2004, AJ, 127, 2948

Woitke, P., \& Helling, Ch. 2004, A\&A, 414, 335 
Table 1: EFFECTIVE TEMPERATURES

\begin{tabular}{llclllllll}
\hline \hline Object & Sp. type & $J-K^{\mathrm{a}}$ & $J-K^{\mathrm{b}}$ & $d(\mathrm{pc})^{\mathrm{c}}$ & $T_{\text {eff }}\left(T_{\text {cr }}\right)^{\mathrm{d}}$ & $T_{\text {eff }}\left(T_{\text {cr }}\right)^{\mathrm{e}}$ & $T_{\text {eff }}{ }^{\mathrm{f}}$ \\
\hline 2MASS 1711+22 & L6.5 & - & 2.25 & 30.20 & $1800 \mathrm{~K}(1800 \mathrm{~K})$ & $1300 \mathrm{~K}(1700 \mathrm{~K})$ & $1545 \mathrm{~K}$ \\
2MASS 1523+30 & L8 & 1.60 & 1.65 & 17.45 & 1500 & $(1800)$ & $1300(1700)$ & 1330 \\
SDSS 1254-01 & T2 & 0.82 & 0.96 & 13.21 & 1300 & $(1800)$ & 1300 & $(1800)$ & 1361 \\
SDSS 1750+17 & T3.5 & 0.12 & 0.83 & 27.59 & 1100 & $(1800)$ & 1300 & $\left(T_{\text {cond }}\right)$ & 1478 \\
\hline
\end{tabular}

${ }^{a}$ MKO system (Knapp et al. 2004)

${ }^{b}$ CIT system (Vrba et al. 2004)

${ }^{c}$ distance based on the parallaxes by Vrba et al.(2004)

${ }^{d}$ based on the analysis of the spectra with UCMs of the uniform value $T_{\mathrm{cr}}=1800 \mathrm{~K}$ (Paper II)

${ }^{e}$ based on the analysis of the absolute fluxes with UCMs of $T_{\text {cr }}$ inferred from the infrared colors (this paper)

${ }^{f}$ based on the bolometric luminosity (Vrba et al. 2004) 

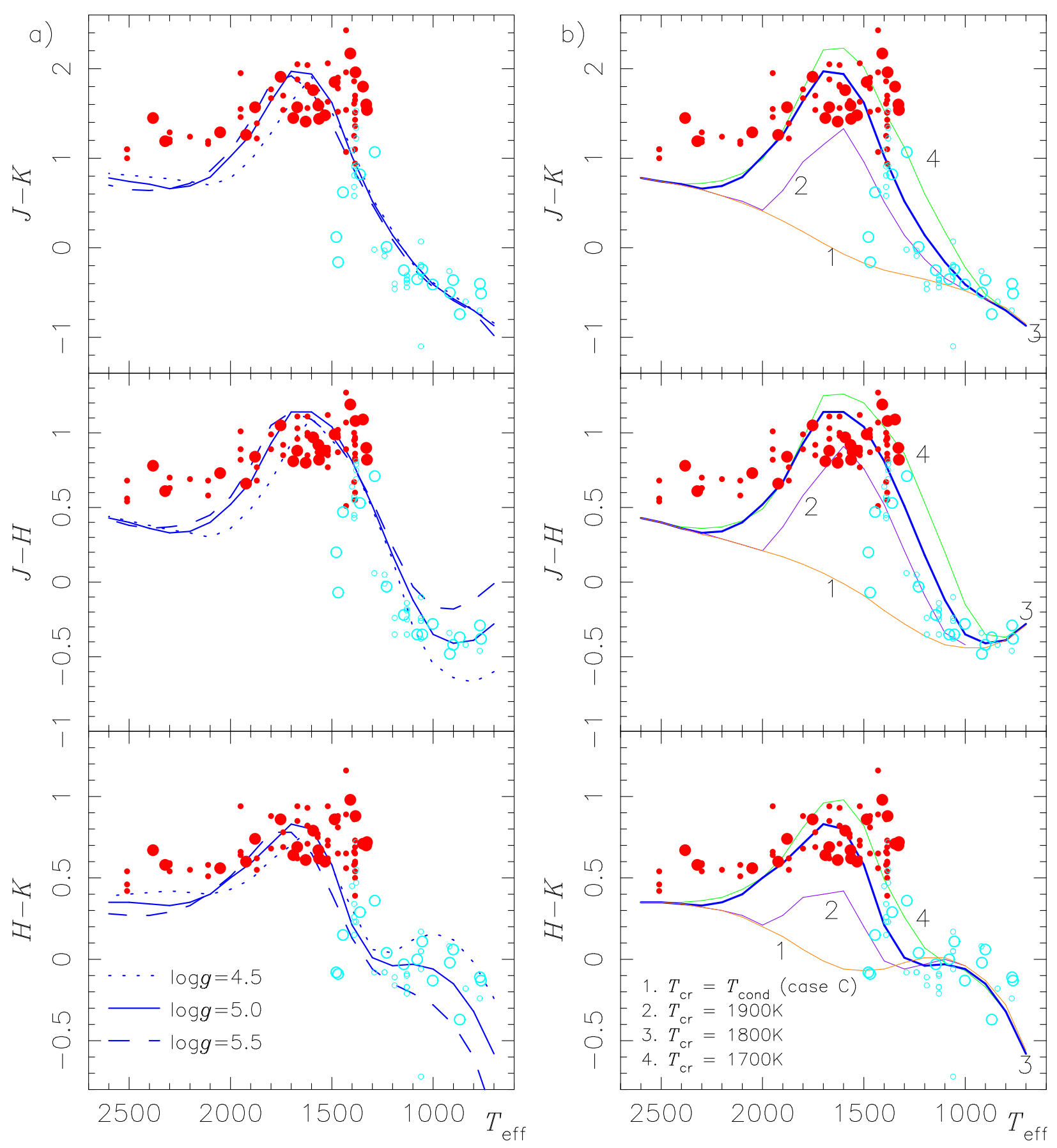

Fig. 1. - Infrared colors on the MKO system (Knapp et al. 2004) are plotted against the effective temperatures (Vrba et al. 2004) by the filled and open circles for L and T dwarfs, respectively. The large and small circles indicates that $T_{\text {eff }}$ values are determined directly from the measured bolometric luminosities and indirectly by the use of $T_{\text {eff }}-$ Sp. Type calibration by Vrba et al.(2004), respectively. $J-K, J-H$, and $H-K$ are shown in the upper, middle, and lower panels, respectively. a) Predicted colors based on the UCMs with $\log g=4.5,5.0$ and 5.5 are shown by the dotted, solid, and dashed lines, respectively $\left(T_{\mathrm{cr}}=\right.$ $1800 \mathrm{~K}$ throughout). b) Predicted colors based on the UCMs with $T_{\text {cr }}=T_{\text {cond }}$ (case C), 1900, 1800 , and $1700 \mathrm{~K}$ are shown by the lines labeled with $1,2,3$, and 4 , respectively. $(\log g=$ $5.0, v_{\text {micro }}=1 \mathrm{~km} \mathrm{~s}^{-1}$ and the solar metallicity throughout). 


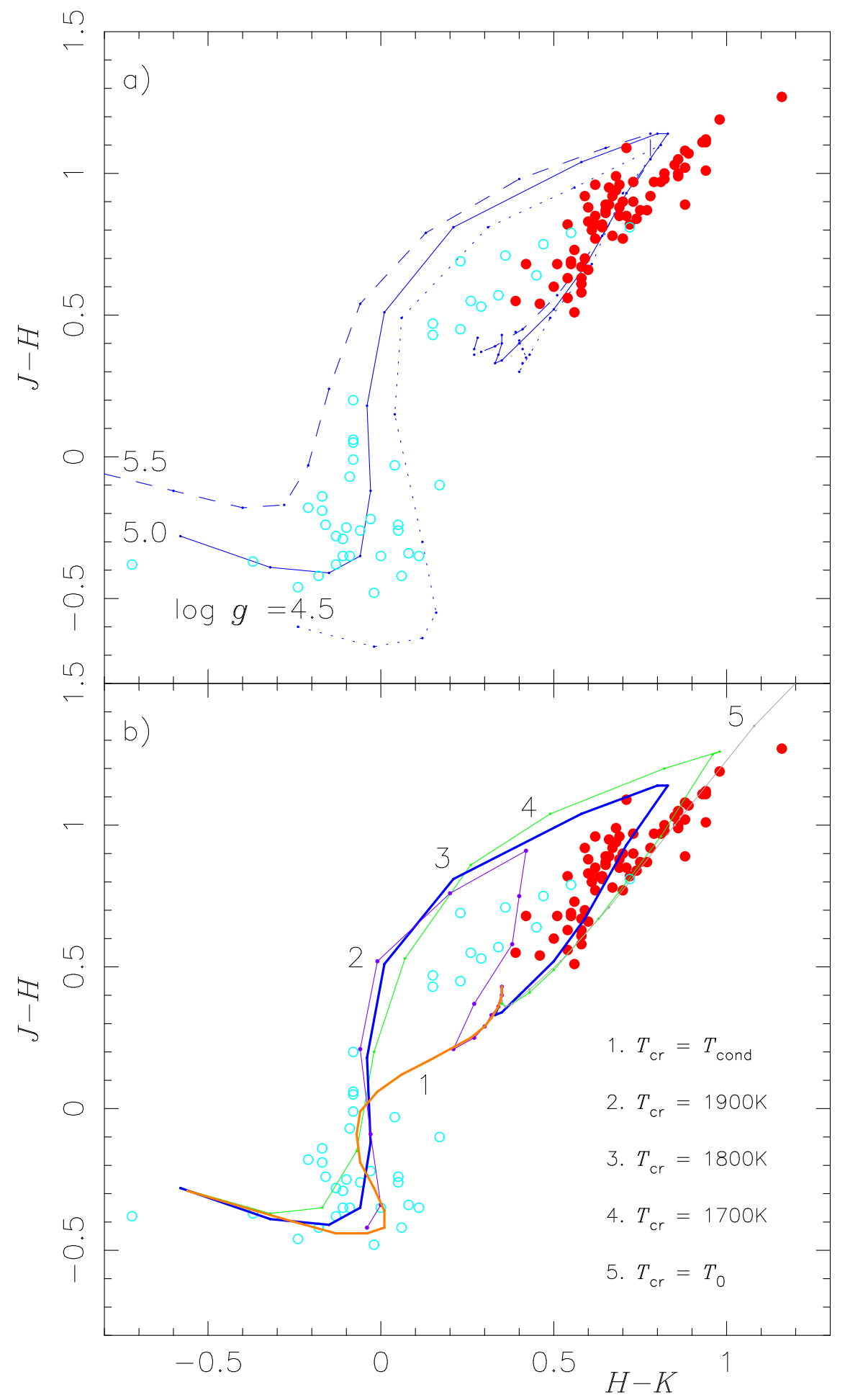

Fig. 2.- Observed $J-H$ vs. $H-K$ on the MKO system (Knapp et al.2004) are shown by the filled and open circles for $\mathrm{L}$ and $\mathrm{T}$ dwarfs, respectively. a) Predicted $J-H$ vs. $H-K$ based on the UCMs with $\log g=4.5,5.0$, and 5.5 are shown by the dotted, solid, and dashed lines, respectively, $\left(T_{\text {eff }}=700-2600 \mathrm{~K}\right.$ and $T_{\text {cr }}=1800 \mathrm{~K}$ throughout $)$. b) Predicted $J-H$ vs. $H-K$ based on the UCMs with $T_{\text {cr }}=T_{\text {cond }}$ (case C), $1900 \mathrm{~K}, 1800 \mathrm{~K}, 1700 \mathrm{~K}$, and $T_{0}$ (case B) are shown by the lines labeled with $1,2,3,4$, and 5 , respectively. $\left(T_{\text {eff }}=700-2600 \mathrm{~K}\right.$ and $\log g=5.0$ throughout). 


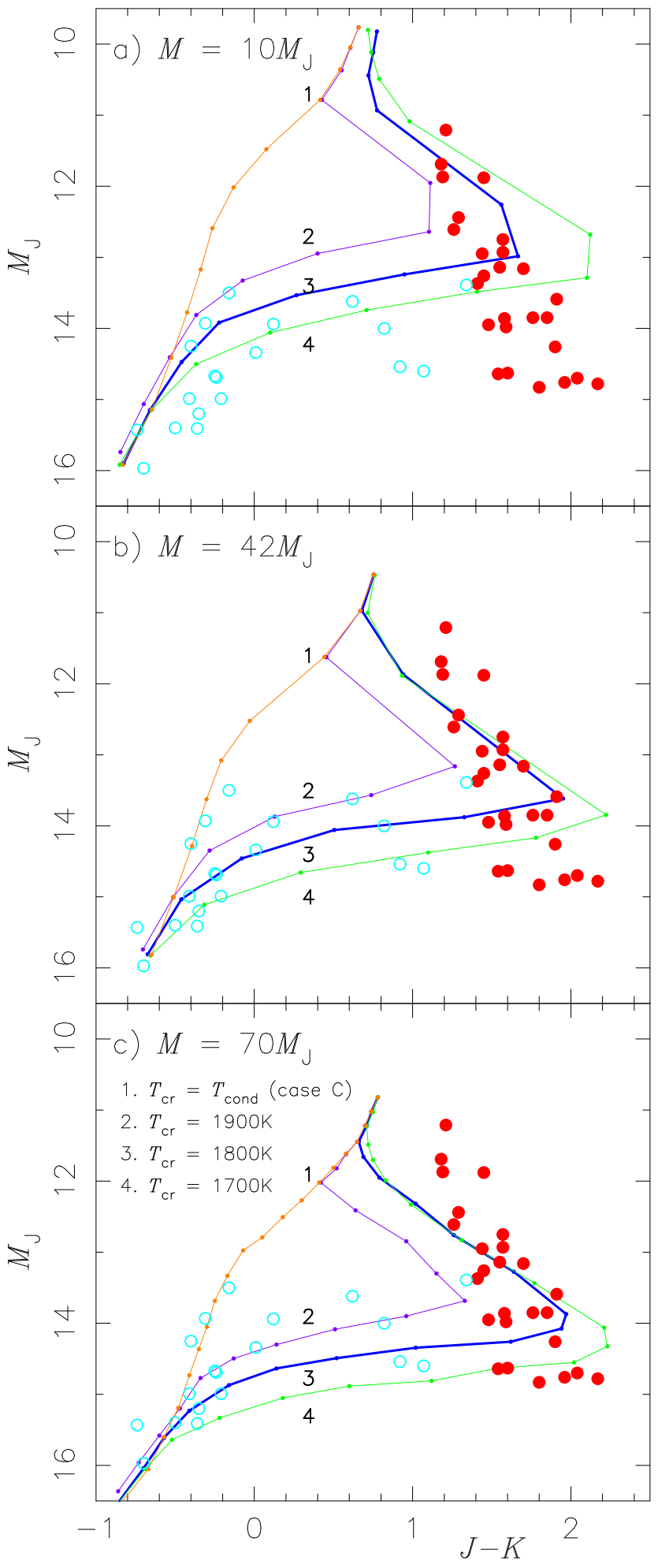

Fig. 3.- Observed $M_{J}$ vs. $J-K$ on the MKO system (Knapp et al.2004) are shown by the filled and open circles for L and T dwarfs, respectively. Predicted $M_{J}$ vs. $J-K$ transformed from the evolutionary tracks by Burrows et al. (1997) via the UCMs with $T_{\text {cr }}=T_{\text {cond }}$, $1900 \mathrm{~K}, 1800 \mathrm{~K}$, and $1700 \mathrm{~K}$ are shown by the lines labeled with 1, 2, 3, and 4, respectively. a) $M=10 M_{\text {Jupiter }}$, b) $M=42 M_{\text {Jupiter }}$, and c) $M=70 M_{\text {Jupiter }} .\left(T_{\text {eff }}=700-2600 \mathrm{~K}\right.$ and $\log g=5.0$ throughout). 


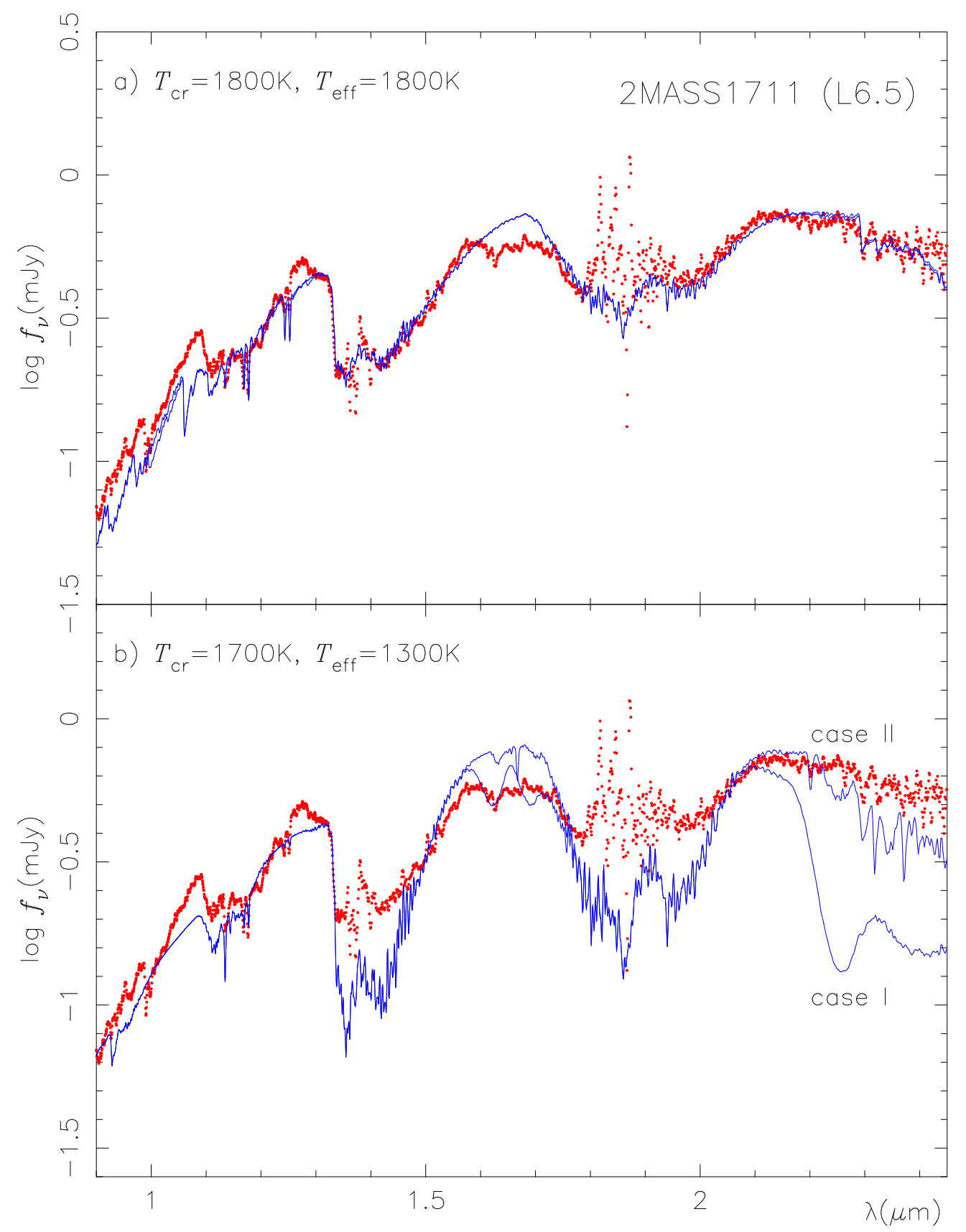

Fig. 4.- The observed spectrum of 2MASS 1711 (L6.5) shown by the dots is compared with the predicted ones based on the UCMs of: a) $T_{\text {cr }}=1800 \mathrm{~K}, T_{\text {eff }}=1800 \mathrm{~K}$, and $\log g=5.0$. b) $T_{\text {cr }}=1700 \mathrm{~K}, T_{\text {eff }}=1300 \mathrm{~K}$, and $\log g=5.0$. 


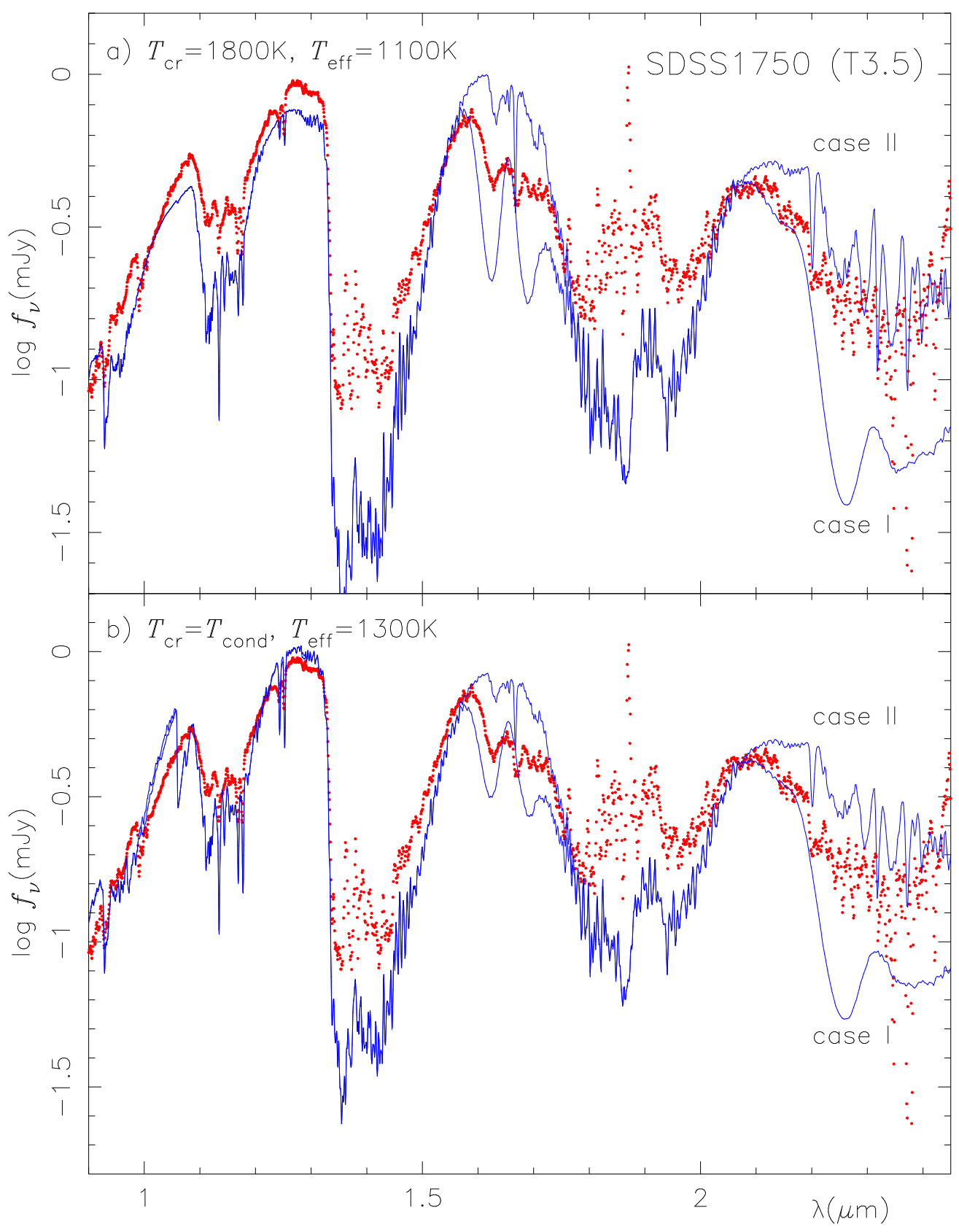

Fig. 5.- The observed spectrum of SDSS 1750 (T3.5) shown by the dots is compared with the predicted ones based on the UCMs of: a) $T_{\text {cr }}=1800 \mathrm{~K}, T_{\text {eff }}=1100 \mathrm{~K}$, and $\log g=5.0$. b) $T_{\text {cr }}=T_{\text {cond }}$ (case C), $T_{\text {eff }}=1300 \mathrm{~K}$, and $\log g=5.0$. 


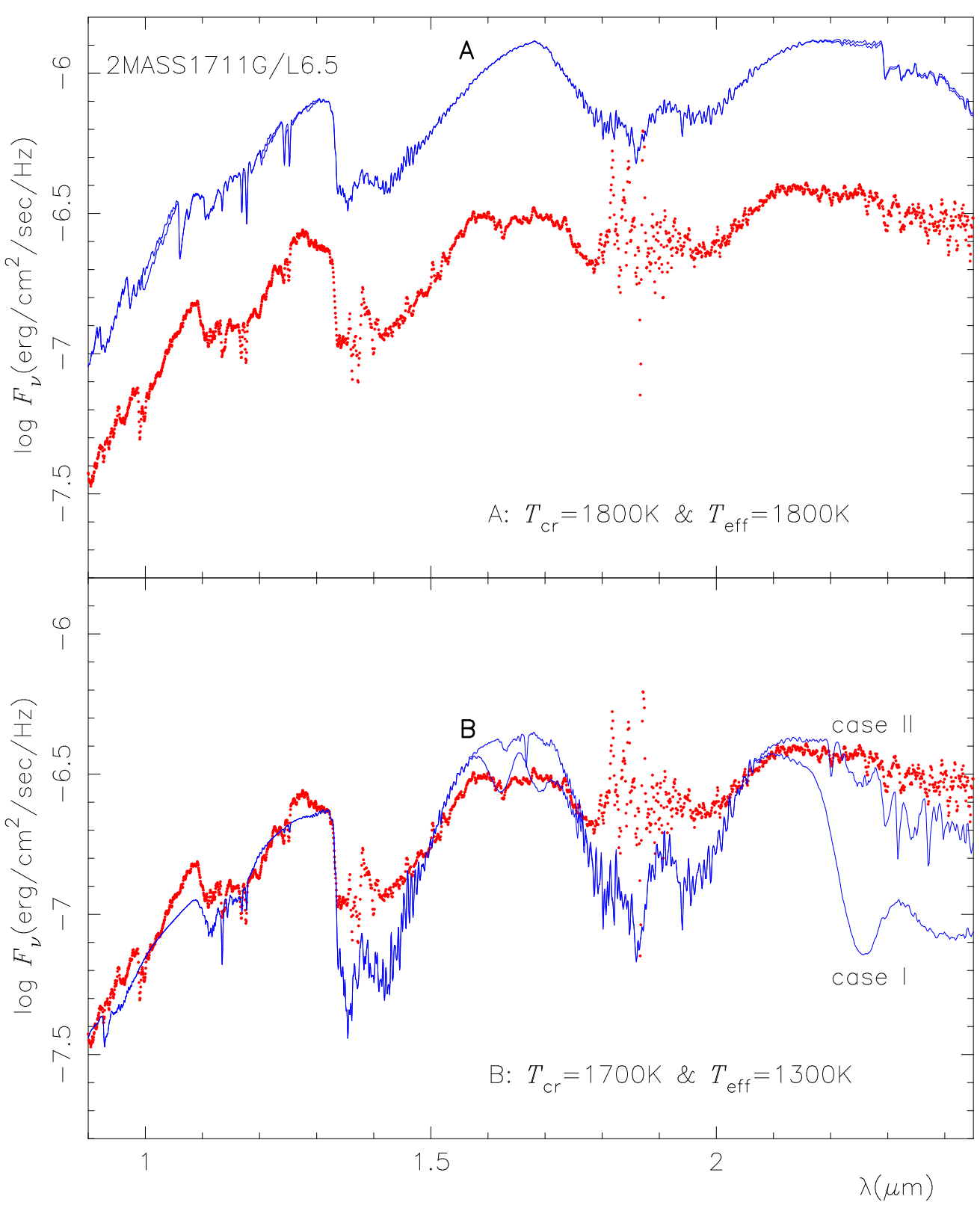

Fig. 6. - The observed spectrum of 2MASS 1711 (L6.5) reduced to the emergent flux on an absolute scale (in unit of $\mathrm{erg} \mathrm{cm}^{-2} \mathrm{sec}^{-1} \mathrm{~Hz}^{-1}$ ) is shown by the dots. The predicted spectrum based on the UCM with $T_{\text {cr }}=1800 \mathrm{~K}, T_{\text {eff }}=1800 \mathrm{~K}$, and $\log g=5.0$ (cf. Fig.6 of Paper II) is shown by the solid line marked with $\mathrm{A}$. Although the observed and predicted spectra can be fitted on the relative scale (i.e. by the shapes of the spectra as in Fig.4), they cannot be fitted on the absolute scale. Infrared colors suggest a lower $T_{\mathrm{HR}}$ for this object(Table 1; Fig. 1b), and the predicted spectrum based on the UCM with $T_{\text {cr }}=1700 \mathrm{~K}, T_{\text {eff }}=1300 \mathrm{~K}$, and $\log g=5.0$ is shown by the solid line marked with B. The observed and predicted spectra can be fitted on the absolute scale, but the predicted water bands appear to be too strong. 


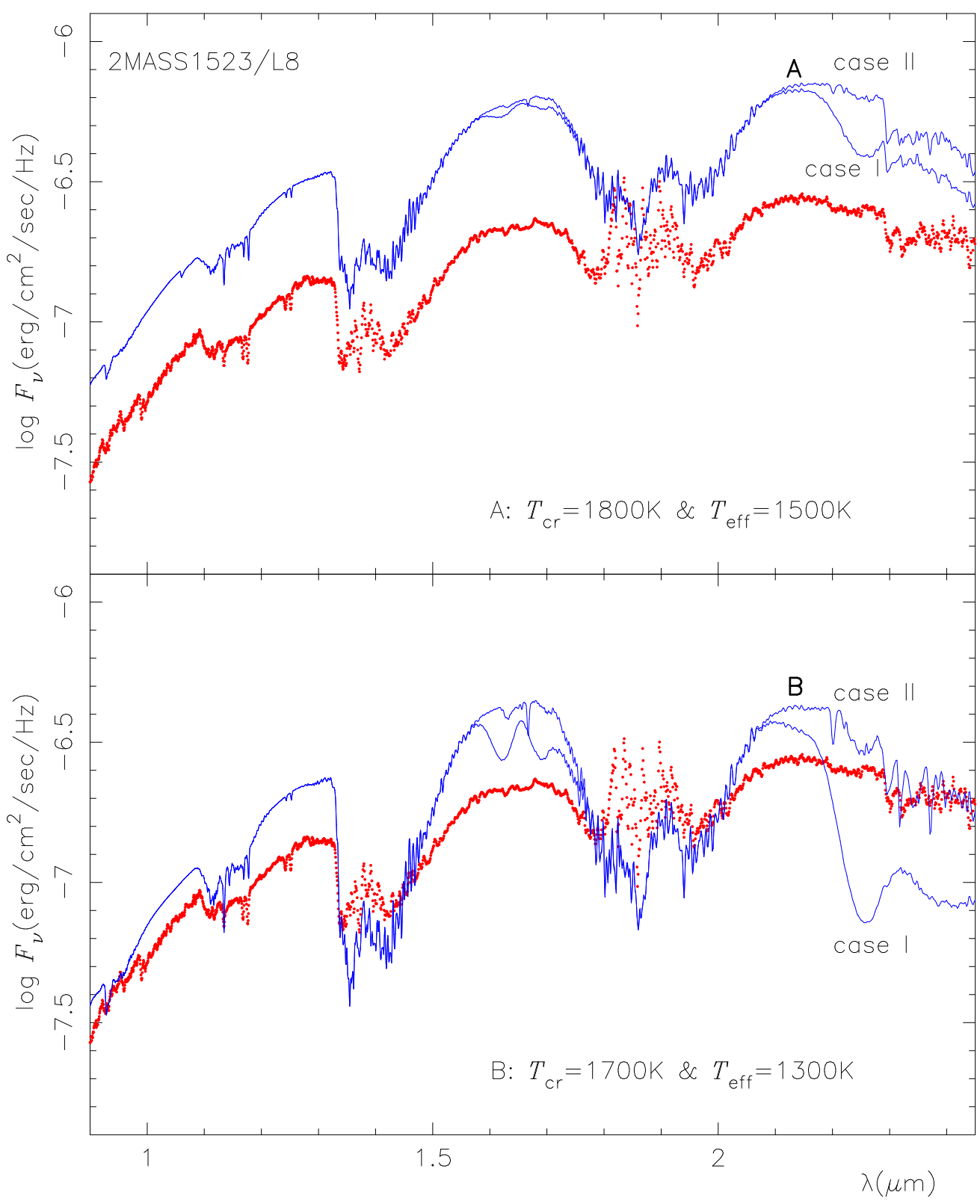

Fig. 7.- The observed spectrum of 2MASS 1523 (L8) reduced to the emergent flux on an absolute scale is shown by the dots. The predicted spectrum based on the UCM with $T_{\text {cr }}=1800 \mathrm{~K}, T_{\text {eff }}=1500 \mathrm{~K}$, and $\log g=5.0$ is shown by the solid line marked with A. Although the observed and predicted spectra can be fitted on the relative scale (Fig.8 of Paper II), it is clear that they cannot be fitted on the absolute scale. Infrared colors suggest a lower $T_{\text {cr }}$ for this object (Table 1; Fig. 1b), and the predicted spectrum based on the UCM with $T_{\text {cr }}=1700 \mathrm{~K}, T_{\text {eff }}=1300 \mathrm{~K}$, and $\log g=5.0$ is shown by the solid line marked with B. The observed and predicted spectra cannot be fitted on the absolute scale as well, but it is to be noted that the uncertainty of the absolute scale is about $0.2-0.3$ dex. 


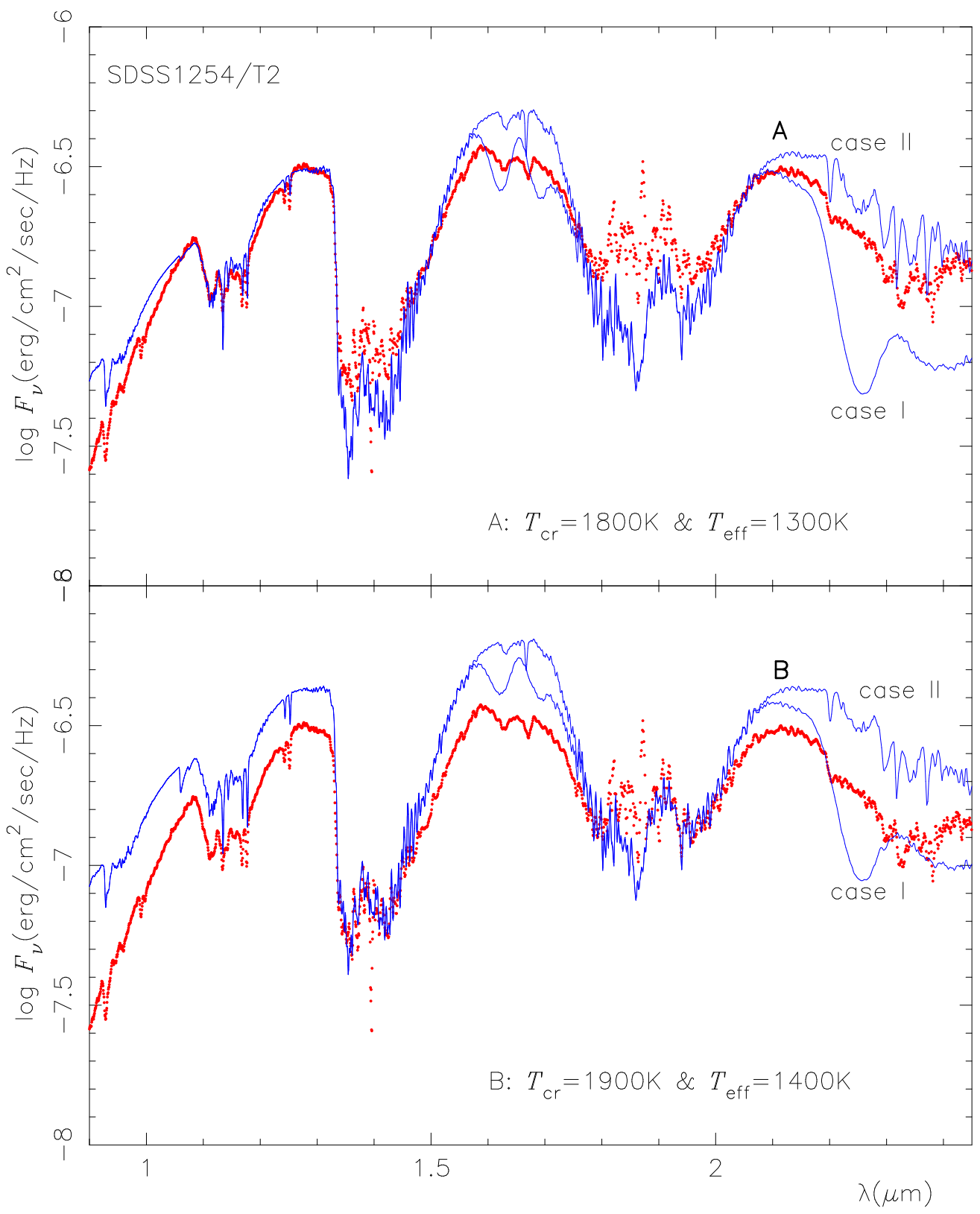

Fig. 8. - The observed spectrum of SDSS 1254 (T2) reduced to the emergent flux on an absolute scale is shown by the dots. The predicted spectrum based on the UCM with $T_{\text {cr }}=1800 \mathrm{~K}, T_{\text {eff }}=1300 \mathrm{~K}$, and $\log g=5.0$ is shown by the solid line marked with A. The observed and predicted spectra agree well both on the relative and absolute scales. Infrared colors suggest $T_{\text {cr }} \approx 1800 \mathrm{~K}$ for this object(Table 1; Fig. 1b), and this also supports the analysis of Paper II (Fig.9). For comparison, the predicted spectrum based on the UCM with $T_{\text {cr }}=1900 \mathrm{~K}, T_{\text {eff }}=1400 \mathrm{~K}$, and $\log g=5.0$ is shown by the solid line marked with B. The curves $\mathrm{A}$ and $\mathrm{B}$ show quite similar and the degeneracy of $T_{\mathrm{cr}}$ and $T_{\text {eff }}$ cannot be removed on the spectra without absolute calibration. 


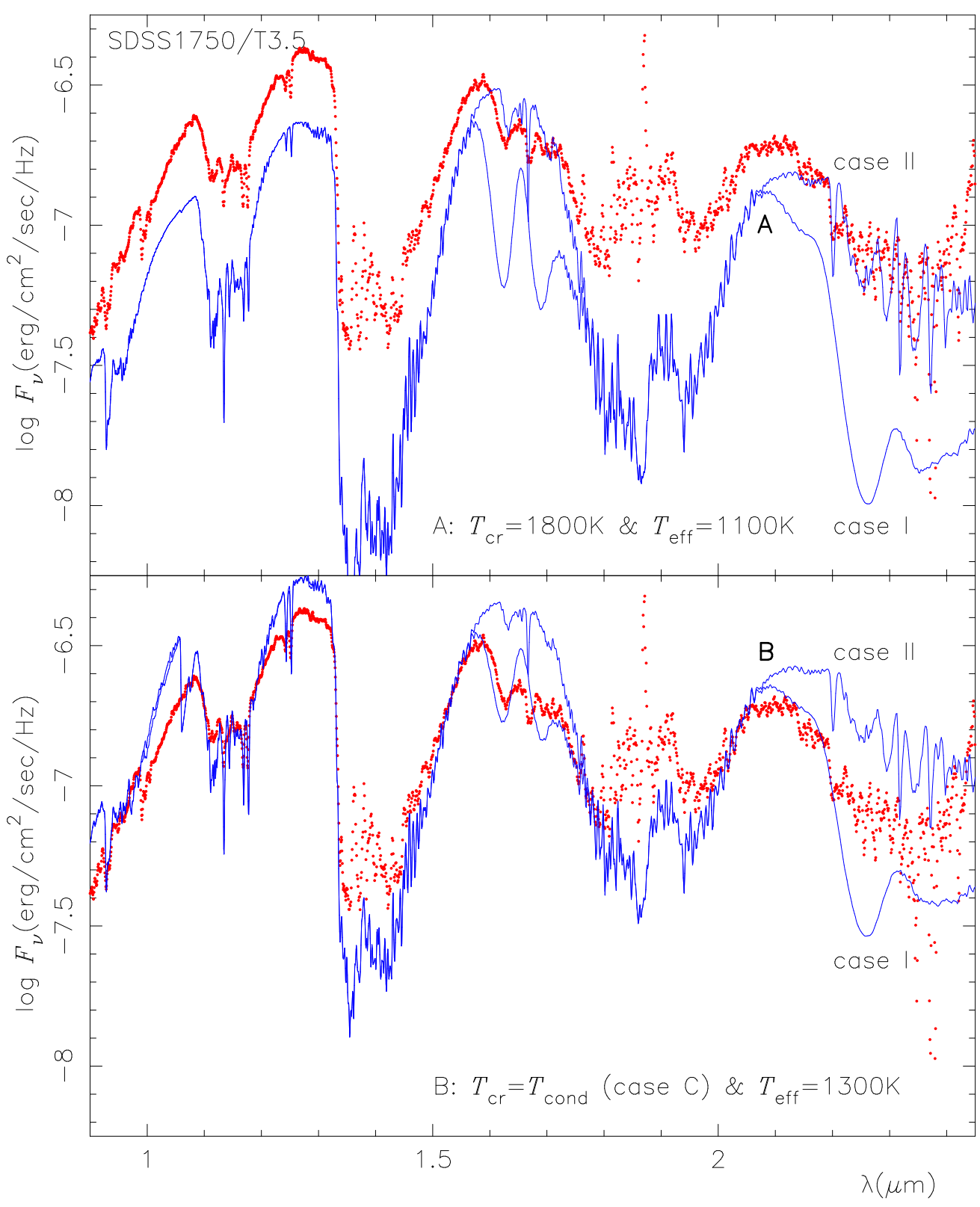

Fig. 9.- The observed spectrum of SDSS 1750 (T3.5) reduced to the emergent flux on an absolute scale is shown by the dots. The predicted spectrum based on the UCM with $T_{\text {cr }}=1800 \mathrm{~K}, T_{\text {eff }}=1100 \mathrm{~K}$, and $\log g=5.0$ is shown by the solid line marked with A. The observed and predicted spectra agree marginally within the possible uncertainty of the absolute scale, although the predicted water bands appear to be too strong (Fig.10 of Paper II). Infrared colors suggest $T_{\text {cr }} \approx T_{\text {cond }}$ (case C) for this object (Table $1 ;$ Fig. 1b), and the predicted spectrum based on the UCM with $T_{\text {cr }}=T_{\text {cond }}$ (case C), $T_{\text {eff }}=1300 \mathrm{~K}$, and $\log g=$ 5.0 is shown by the solid line marked with B. The observed and predicted spectra now show better agreement both on the absolute and relative scales. 


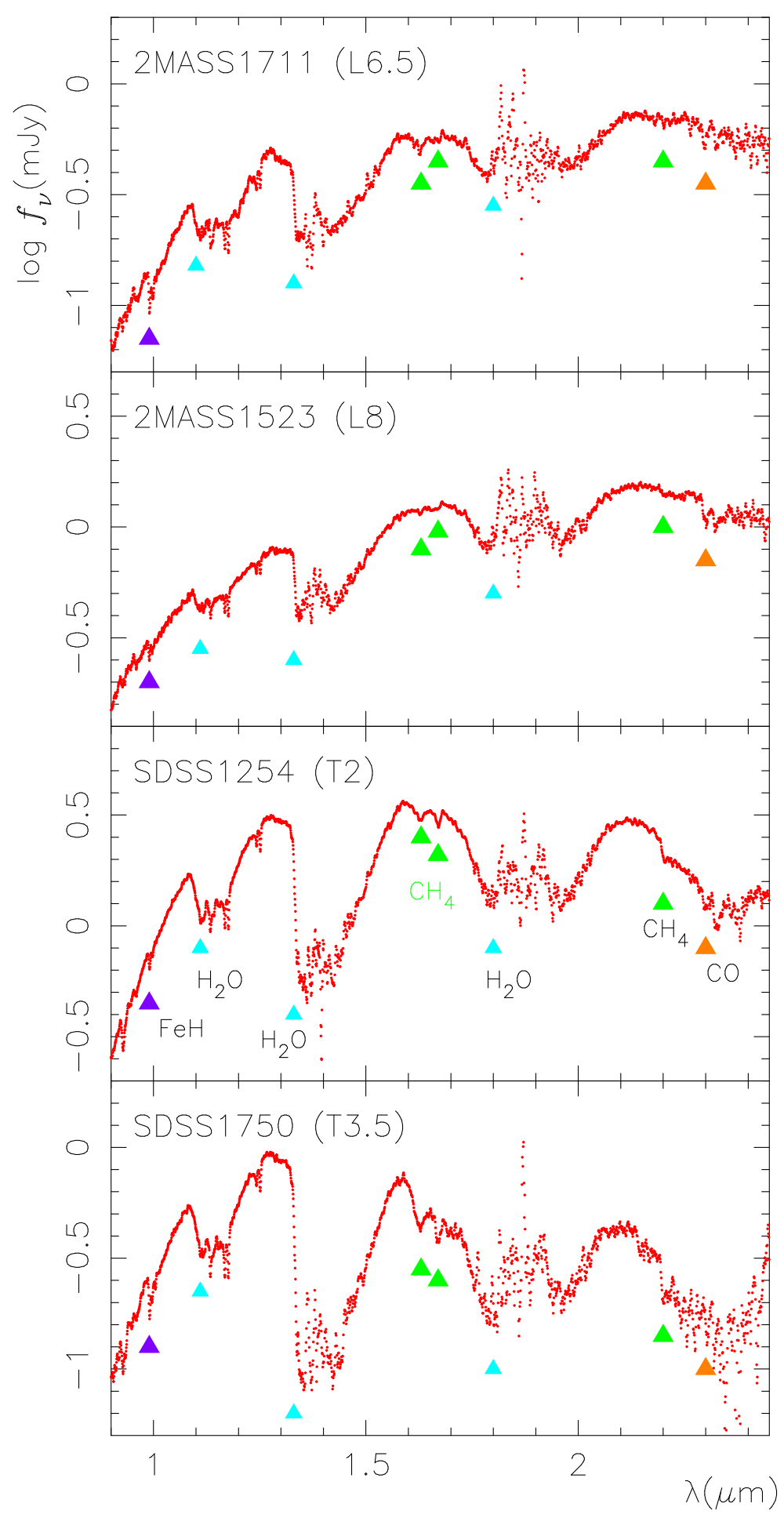

Fig. 10. - The observed spectral sequence between L6.5 and T3.5 are summarized. These objects were interpreted as a temperature sequence $\left(T_{\text {eff }}\right.$ from $1800 \mathrm{~K}$ to $\left.1100 \mathrm{~K}\right)$ based on the UCMs with an uniform value of $T_{\text {cr }}=1800 \mathrm{~K}$ (Paper II). The same observed data reduced to an absolute scale are reanalyzed again with the UCMs, but freed from the assumption of the uniform value of $T_{\mathrm{cr}}$, and the result revealed that the values of $T_{\text {eff }}$ for all these objects are about the same at $1300 \mathrm{~K}$ while the values of $T_{\text {cr }}$ should increase (or the thickness of the dust cloud decreases) from L to $\mathrm{T}$ dwarfs. 


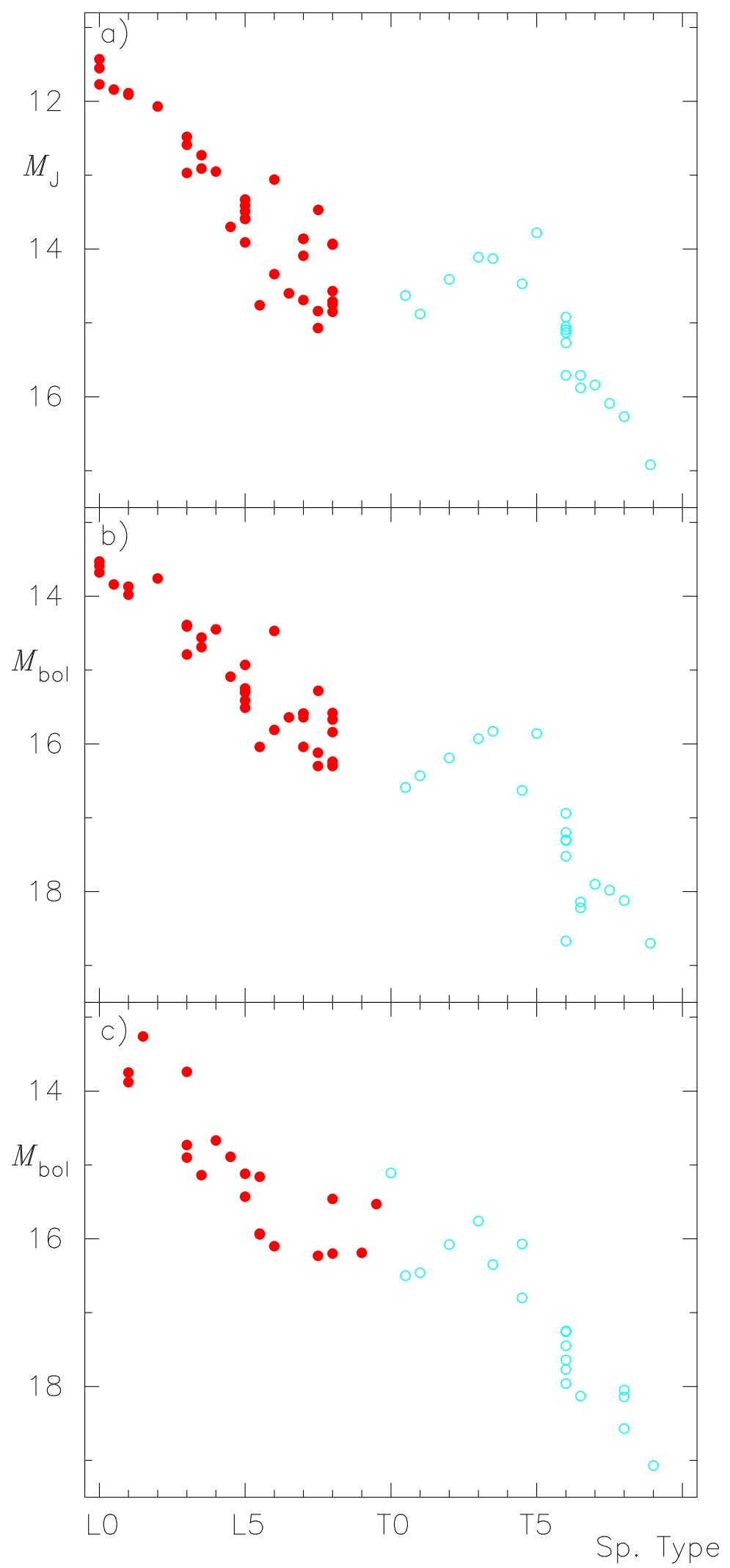

Fig. 11.- - a) $M_{J}$ plotted against L-T spectral types based on the data by Vrba et al. (2004). b) $M_{\text {bol }}$ plotted against L-T spectral types based on the data by Vrba et al. (2004). c) The same as b) but based on the data by Golimowski et al. (2004). 


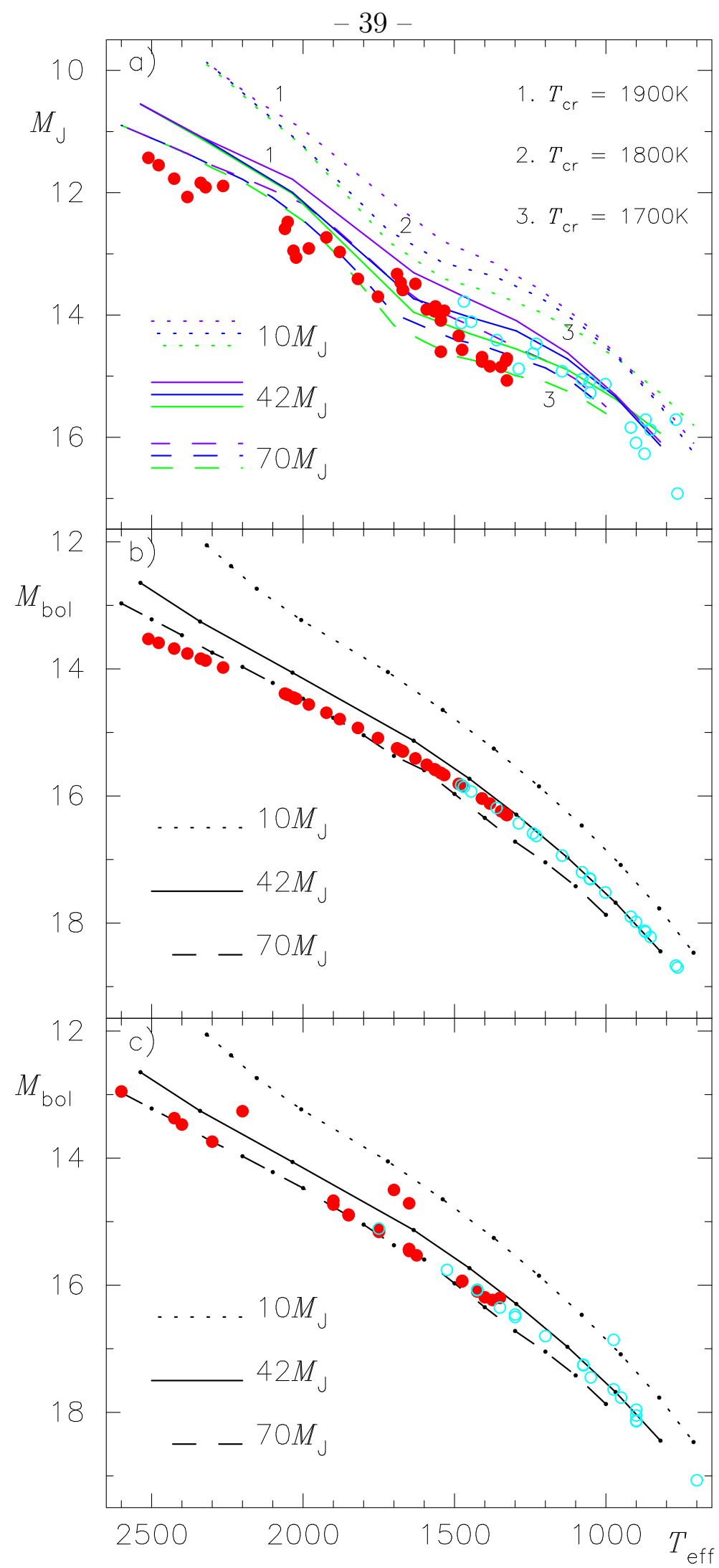

Fig. 12.- a) $M_{J}$ plotted against $T_{\text {eff }}$ based on the data by Vrba et al. (2004). Also $M_{J}$ transformed from $M_{\mathrm{bol}}$ of Burrows et al. (1997) models of $M=10,42$, and $70 M_{\mathrm{Jupiter}}$ via UCMs are overlaid. Note that each case is triplicated for $T_{\text {cr }}=1900,1800$, and $1700 \mathrm{~K}$. b) $M_{\text {bol }}$ plotted against $T_{\text {eff }}$ based on the data by Vrba et al. (2004). The evolutionary tracks for $M=10,42$, and $70 M_{\text {Jupiter }}$ by Burrows et al.(1997) are overlaid. c) The same as b) but based on the data by Golimowski et al. (2004) for an age of 3 Gyr. 